\title{
Fast Vesicle Transport Is Required for the Slow Axonal Transport of Synapsin
}

\author{
Yong Tang, ${ }^{1}$ David Scott, ${ }^{1}$ Utpal Das, ${ }^{1}$ Daniel Gitler, ${ }^{2}$ Archan Ganguly, ${ }^{1}$ and Subhojit Roy ${ }^{1,3}$ \\ ${ }^{1}$ Department of Pathology, University of California, San Diego, La Jolla, California 92093, ${ }^{2}$ Department of Physiology and Cell Biology, Faculty of Health \\ Sciences, and Zlotowski Center for Neuroscience, Ben-Gurion University of the Negev, Beer-Sheva 84105, Israel, and ${ }^{3}$ Department of Neurosciences, \\ University of California, San Diego, La Jolla, California 92093
}

\begin{abstract}
Although it is known that cytosolic/soluble proteins synthesized in cell bodies are transported at much lower overall velocities than vesicles in fast axonal transport, the fundamental basis for this slow movement is unknown. Recently, we found that cytosolic proteins in axons of mouse cultured neurons are conveyed in a manner that superficially resembles diffusion, but with a slow anterograde bias that is energy- and motor-dependent (Scott et al., 2011). Here we show that slow axonal transport of synapsin, a prototypical member of this rate class, is dependent upon fast vesicle transport. Despite the distinct overall dynamics of slow and fast transport, experimentally induced and intrinsic variations in vesicle transport have analogous effects on slow transport of synapsin as well. Dynamic cotransport of vesicles and synapsin particles is also seen in axons, consistent with a model where higher-order assemblies of synapsin are conveyed by transient and probabilistic associations with vesicles moving in fast axonal transport. We posit that such dynamic associations generate the slow overall anterogradely biased flow of the population ("dynamic-recruitment model”). Our studies uncover the underlying kinetic basis for a classic cytosolic/soluble protein moving in slow axonal transport and reveal previously unknown links between slow and fast transport, offering a clearer conceptual picture of this curious phenomenon.
\end{abstract}

\section{Introduction}

Previous pulse-chase radiolabeling experiments have established that perikaryally synthesized proteins are delivered into axons and synapses via fast and slow axonal transport (Lorenz and Willard, 1978; Black and Lasek, 1980). Whereas fast transport carries membrane-spanning/anchoring proteins in vesicles that move rapidly at $\sim 50-400 \mathrm{~mm} / \mathrm{d}(0.5-4 \mu \mathrm{m} / \mathrm{s})$, slow transport carries the principal cytoskeletal elements tubulin and neurofilament proteins moving at $\sim 0.02-0.1 \mathrm{~mm} / \mathrm{d}(0.002-0.01 \mu \mathrm{m} / \mathrm{s})$, as well as hundreds of diverse cytosolic/soluble proteins, generally moving slightly faster, at overall rates of $\sim 1-10 \mathrm{~mm} / \mathrm{d}(0.01-0.1 \mu \mathrm{m} / \mathrm{s}$ ) (for review, see Lasek et al., 1984). The two latter groups are also called slow component $\mathrm{a}$ and $\mathrm{b}$ (SCa and $\mathrm{SCb}$, respectively). The rate class $\mathrm{SCb}$ includes proteins involved in presynaptic functioning (synapsin, clathrin, $\alpha$-synuclein, CaMKIIa), axonal growth/maintenance (metabolic enzymes, chaperones), motors (dynein, myosin), and also some cytoskeletal/cytoskeleton-associated proteins (actin, spectrin, cofilin, tau) (Brady et al., 1981; Garner and Lasek, 1982; Lasek et

Received March 15, 2013; revised Aug. 12, 2013; accepted Aug. 14, 2013.

Author contributions: Y.T., D.S., U.D., D.G., and S.R. designed research; Y.T., D.S., U.D., and A.G. performed research; Y.T., D.S., U.D., and S.R. analyzed data; Y.T., D.S., and S.R. wrote the paper.

This work was supported by National Institutes of Health Grant R01NS075233 and the March of Dimes (Basil 0 Connor) to S.R. We thank Drs. George Patterson and Jennifer Lippincott-Schwartz (National Institutes of Health) for the PAGFP construct; and Drs. George Augustine (Duke University), Leon Laganado (MRC, Cambridge, United Kingdom), and Martha Vaughan (National Institutes of Health) for the GFP:synapsin-la, synaptophysin:mRFP, and ARF-1 constructs, respectively.

The authors declare no competing financial interests.

Correspondence should be addressed to Dr. Subhojit Roy, University of California, San Diego, 9500 Gilman Drive, BSB 1030A, MC0612, La Jolla, CA 92093. E-mail: s1roy@ucsd.edu.

DOI:10.1523/JNEUROSCI.1148-13.2013

Copyright $\odot 2013$ the authors $\quad 0270-6474 / 13 / 3315362-14 \$ 15.00 / 0$ al., 1984; Baitinger and Willard, 1987; Bray et al., 1992; Mercken et al., 1995; Dillman et al., 1996; Yuan et al., 1999; Lund and McQuarrie, 2001; Bourke et al., 2002). Despite this compositional diversity, the overall movement of diverse cytosolic/soluble/SCb proteins seems surprisingly cohesive (Garner and Lasek, 1982).

Although radiolabeling experiments defined the various rate classes, they were descriptive by design and do not provide much insight into the molecular mechanisms that generate this motion. In recent years, with the development of fluorescent vectors and new live imaging tools, it is clear that the overall fast movement seen in radiolabeling studies is generated by a rapid and persistent vectorial flux of numerous individual vesicles that bind to motors, and many molecular details of this process are known (for review, see Vale, 2003; Verhey and Hammond, 2009). Similarly, substantial evidence supports the notion that neurofilament proteins move as assembled polymers (Roy et al., 2000; Wang et al., 2000; Brown et al., 2005). However, despite advances in our knowledge of vesicular and cytoskeletal protein transport, basic mechanisms generating the slow transit of hundreds of soluble/ cytosolic proteins in SCb have remained mysterious.

A major obstacle in the slow-transport field has been a lack of methods that can visualize the phenomenon (Brown, 2003), particularly true for cytosolic proteins where inherent solubility makes optical imaging challenging. Recently, we used photoactivation-based imaging strategies to visualize dynamics of "pulse-labeled" cytosolic proteins (Roy et al., 2011; Scott et al., 2011). We found that cytosolic cargoes move as unusual biased "streams" of fluorescence that are motor- and microtubuledependent, and we proposed a working model where soluble protein assemblies are conveyed by transiently associating with 
persistently moving hypothetical structures we tentatively termed "mobile units" (Scott et al., 2011; Tang et al., 2012a). Using synapsin-1a as an exemplary SCb protein (referred to as "synapsin" throughout the text); here we provide evidence that the "mobile units" are vesicles moving in fast axonal transport. Using a combination of cell biology and modeling, our data offer a much clearer conceptual picture of cytosolic cargoes as they emerge from the soma and are transported into axons and synapses.

\section{Materials and Methods}

Cell culture, transfections, plasmids, and antibodies. Hippocampal cultures were obtained from brains of postnatal (P0-P2) CD-1 mice (either sex) and maintained as previously described (Roy et al., 2011; Scott et al., 2011). Neurons were transfected with the appropriate fluorescent constructs at 7-9 DIV with Lipofectamine-2000 (Invitrogen) and allowed to express for the times indicated in the text. For the overall transport/ targeting experiments in Figures 1 and 2, neurons were imaged 4-5 h after adding the DNA/Lipofectamine mixture, using the protocols described in Figure 1A. All axonal transport studies in subsequent figures were performed $\sim 17-20 \mathrm{~h}$ after transfection. All animal studies were performed in accordance with University of California guidelines. Constructs were obtained from various laboratories as noted in Acknowledgments. DsRed-mitochondria was obtained from Clontech. Brefeldin A (BFA; Sigma) was stored as a $5 \mathrm{mg} / \mathrm{ml}$ stock solution in ethanol at $-20^{\circ} \mathrm{C}$ and subsequently diluted in imaging medium for a final concentration of $1 \mu \mathrm{g} / \mathrm{ml}$. Ethanol alone was used as controls in all BFA experiments.

Microscopy and live imaging. All live imaging experiments were performed using an Olympus IX81 inverted motorized epifluorescence microscope equipped with CoolSNAP $H^{2}$ cameras (Photometrics). Details of the photoactivation setup are described in a methods paper (Roy et al., 2011). Briefly, a dual-source light illuminator (IX2-RFAW, Olympus) was used to simultaneously visualize and photoactivate a discrete ROI within the axon. Kymographs were generated using dropdown menus in MetaMorph. Immediately before live imaging, neurons were transferred to Hibernate media (Brainbits), supplemented with $2 \%$ B27, 2 mm Glutamax, $0.4 \%$ D-glucose, $37.5 \mathrm{~mm} \mathrm{NaCl}$ (Roy et al., 2011; Scott et al., 2011), and maintained at $37^{\circ} \mathrm{C}$ (Precision Control Weatherstation) for the duration of the experiments. Axons were identified based on morphology, and only neurons with unambiguous morphology were selected for imaging (Roy et al., 2011; Scott et al., 2011). For photoactivation experiments, PAGFP:synapsin was photoactivated for $1 \mathrm{~s}$ and imaged at 2 frames/s with an $100 \times$ oil-immersion lens unless otherwise stated. Synaptophysin:GFP/mRFP transport was imaged using the stream acquisition function (MetaMorph) at 5 frames/s and $200 \mathrm{~ms}$ continuous exposure (with no time interval between images). The field diaphragm was closed down to expose only the portion of the axon that was being imaged, and this greatly minimized photobleaching of the rapidly moving particles. Simultaneous imaging of GFP/RFP fluorophores was performed using a dual cam system (Photometrics) as described previously (Scott et al., 2011). The relatively high frame rates and short total imaging times generated simple kymographs that could be manually analyzed. All transport imaging was performed in primary axons emerging from the soma (not branches), and kymographs were analyzed by an observer blinded to the experimental conditions (for further details on our parameters for imaging vesicle transport, see Tang et al., 2012b).

Image analysis-I: photoactivation and "tandem-imaging" experiments. The intensity-center assay was performed using algorithms written in MATLAB (MathWorks) as described previously (Roy et al., 2011, Tang et al., 2012a). Briefly, after photoactivation and time-lapse imaging, the movies were background-subtracted and the photoactivated ROI was cropped. Intensity line scans along the axon were generated for each frame in the movie, and the maximum intensity point ("intensity center") was calculated. Shifts in the fluorescence population resulted in a corresponding shift of the intensity center as well, and these were numerically plotted in Prism (GraphPad) for display. For long-term imaging of vesicle transport (see Fig. 5), neurons were transfected with synaptophysin:mRFP and the field diaphragm was closed down to reveal an $\sim 20 \mu \mathrm{m}$
ROI, which was imaged for $300 \mathrm{~s}$. These procedures prevented photobleaching of particles entering the small ROI, allowing stream imaging at 5 frames/s for extended periods. Synaptophysin and synapsin were tandemly imaged in the same axonal ROI as described in Results. For synaptophysin, the "before" and "after" particle counts in the ROI were averaged to obtain an estimate of the moving particles (the numbers were comparable; see Fig. $6 B$ ). For synapsin, average intensity center shifts were obtained as described above.

Image analysis-II: synaptic targeting experiments. DIV-10 neurons cotransfected with GFP-tagged synapsin (or synaptophysin) and untagged mCherry, and were imaged for up to $5 \mathrm{~h}$ at the indicated time compressions to document the overall mobility of slow and fast transport proteins into axons and boutons (see Figs. 1 and 2). $z$-stacks/maximumprojection images were obtained at each time point and stitched together to generate a time lapse. Axons and boutons were initially identified by diffusion of soluble mCherry into these processes and varicosities, as diffusion is very rapid over these short distances. Only boutons that were stable for the entire duration of the acquisition were chosen for quantification. To quantify the fluorescence accumulation, $8 \times 8$ pixel circular regions were drawn around each bouton, and average intensities were obtained for each frame within the time lapse. Fluorescence intensity of a bouton at a given time point $(\mathrm{F})$ was normalized to the fluorescence intensity in the first frame $\left(\mathrm{F}_{0}\right)$ and expressed as a ratio. Axons were traced manually, and distances of synaptic boutons from the soma/axon junction were calculated by algorithms written in MATLAB. Boutons were then binned into four evenly distributed $50 \mu \mathrm{m}$ quartiles based on their distance from the soma (calculated manually), and analyzed as described in Results. Only neurons with unambiguous morphology and showing similar levels of protein expression (based on mCherry fluorescence) were selected for these experiments.

Axonal transport simulations. Technical details regarding generation of the simulations and analyses of the resultant data were described previously (Scott et al., 2011). The basic modeling platform was adapted from a simulated 3D biophysical environment developed by Jonathan B. Alberts, University of Washington, Friday Harbor, WA (ParMspindle, source code available at http://celldynamics.org), used to simulate intracellular movements of heterogeneous particle populations subject to Brownian and collision forces in an aqueous solution. The model consisted of three main simulated components: the axon, the "mobile units" (henceforth called vesicles), and the synapsin particles. (1) Axon: The simulated axon was defined as a $300-\mu \mathrm{m}$-long segment of axon with a radius of $0.25 \mu \mathrm{m}$. (2) Vesicles: Anterograde vesicles were simulated moving on linear paths running proximal to distal along the length of the simulated axon with constant velocities. The velocities of individual mobile units were sampled from a normal distribution with a mean of 1.818 $\mu \mathrm{m}$ and $\mathrm{SD}$ of $0.7427 \mu \mathrm{m} / \mathrm{s}$, and mobile units were synthesized at a rate of $0.4 \mathrm{~Hz}$. The velocity distribution and synthesis frequency were determined empirically from our movies of synaptophysin transport in hippocampal axons. (3) Cytosolic/SCb particles: The diffusion coefficient of GFP:synapsin molecules in cultured hippocampal axons $(1.1 \times$ $10^{-12} \mathrm{~m}^{2} / \mathrm{s}$ ) (Tsuriel et al., 2006) was used to calculate Brownian forces for simulated cytosolic particles. Cytosolic particles were allowed to associate with vesicles upon random collision. Upon such association, cytosolic particles remained bound to vesicles until random Brownian forces exceeded the association strength of the cytosolic particle with the vesicle. Association strengths of individual cytosolic particles with arbitrary mass were sampled from our previous simulations (mean $\pm \mathrm{SD}$ : $0.095 \pm 0.01 \mu \mathrm{m} / \mathrm{ms}^{2}$ ) (Scott et al., 2011). Kymographs were generated retrospectively from the $3 \mathrm{D}$ coordinates of cytosolic particles or vesicles recorded over the course of the simulation. Kymographs were analyzed using bin-center algorithms as described previously (Scott et al., 2011).

\section{Results}

\section{Characterization of overall dynamics of slow and fast component proteins in cultured neurons}

In this manuscript, we compare and contrast the transport behavior of slow and fast axonal transport in our model system using cultured hippocampal neurons, considering both short- 


\section{A Experimental design}
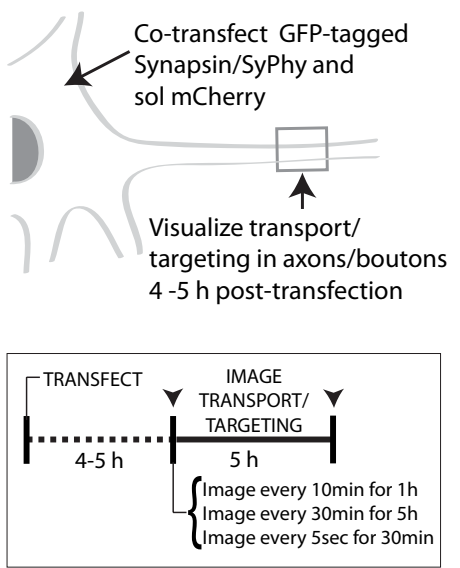

C GFP:Synapsin kymographs

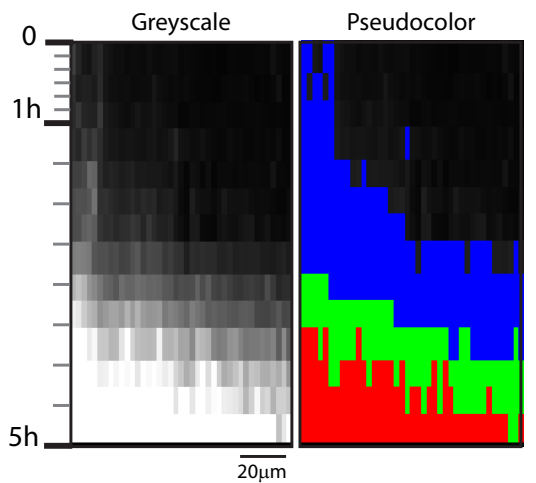

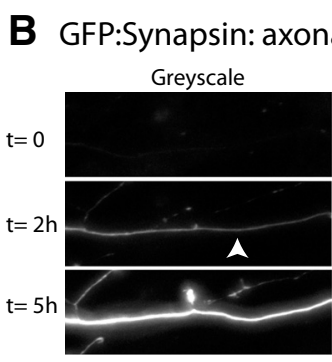
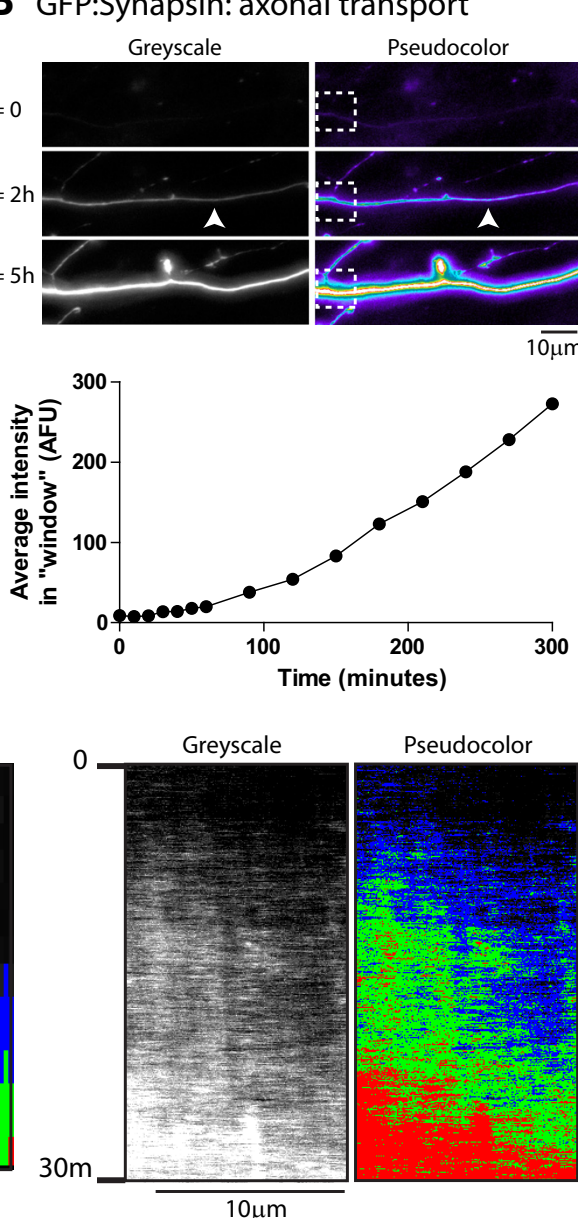

$0 \mu \mathrm{m}$

D mRFP:SyPhy

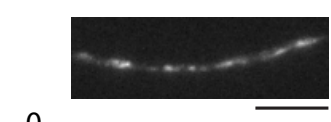

0
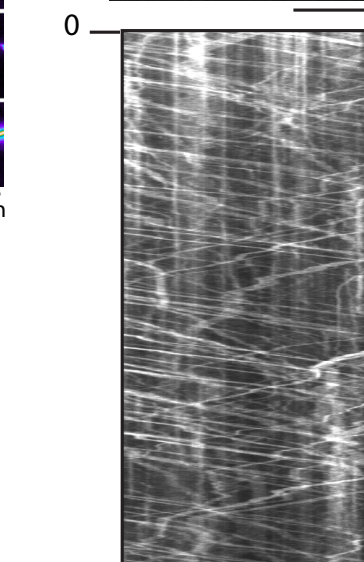

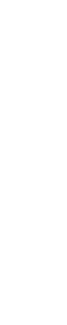

Pseudocolor

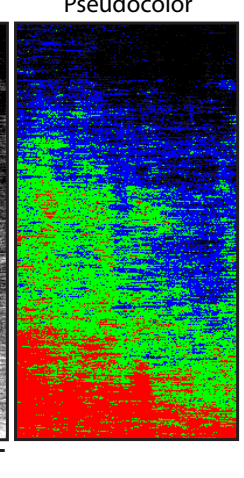

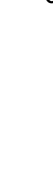

$280 \mathrm{~s}$

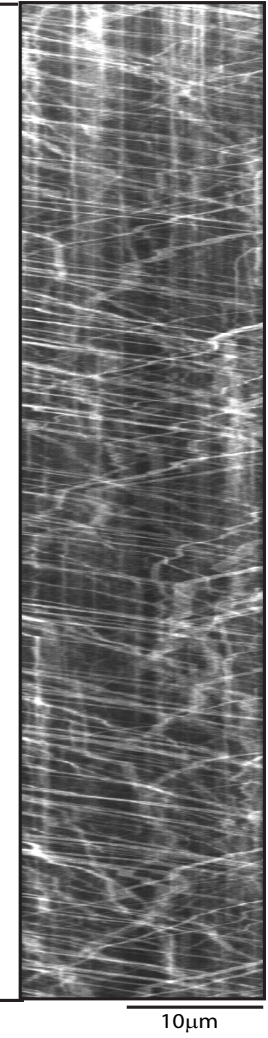

Figure 1. Differential dynamics of slow and fast transport proteins in cultured neurons. $\boldsymbol{A}$, Overall experimental design (also applies to Fig. 2): Cultured hippocampal neurons were cotransfected with GFP-tagged synapsin (or synaptophysin) and soluble mCherry; and their axonal transport/presynaptic accumulation was imaged for up to $5 \mathrm{~h}$ at various time compressions. $\boldsymbol{B}$, Slow axonal transport of synapsin. Selected frames from a time-lapse shows slow ingress of GFP:synapsin into axons over $5 \mathrm{~h}$ (arrowhead indicates "front"). Anterograde is left to right; axon segment shown is $\sim 80 \mu \mathrm{m}$ away from soma/axon junction. Graph below, Rise of fluorescence intensity within the boxed R0I ("window") above. Images were taken every 10 min for $1 \mathrm{~h}$; and then every 30 min for the next 4 h. C, Kymographs from long-term transport imaging of GFP:synapsin, distance is on the $x$-axis, time on $y$-axis. Left panels, Grayscale and pseudocolor kymographs of the axon shown in $\boldsymbol{B}$. There is slow, gradual accumulation of fluorescence in the axon over $5 \mathrm{~h}$ of imaging (each tick on left represents an imaging time point). Right panels, Kymographs from an axon that was imaged with a relatively higher time compression (one image every $5 \mathrm{~s}$ ) to highlight the "wave-like" kinetics of synapsin transport. Regression analysis of the wave-front predicts average rates of $0.02-0.03$ $\mu \mathrm{m} / \mathrm{s}(\sim 1.7-2.5 \mathrm{~mm} / \mathrm{d})$, in line with known SCb rates. D, Experiments with tagged synaptophysin yielded entirely different results, where a plethora of mobile vesicles were seen under all imaging conditions. A kymograph from long-term imaging of mRFP-tagged synaptophysin (mRFP:SyPhy) in axons is shown as an example. Discrete moving vesicles are seen, unlike the slow-moving "wave" seen with synapsin.

term dynamics as well as long-term kinetic behavior of these two rate classes. Accordingly, we first characterized the overall dynamics of two candidate slow and fast transport proteins, synapsin and synaptophysin, respectively, in our cultured neurons. Although overall dynamics of slow and fast cargoes are obviously different in radiolabeling experiments, this distinction has not been demonstrated in cultured neurons. Rather, previous studies in cultured neurons, including our own, have typically visualized slow transport at short time scales and inferred long-term transport behavior. Here we first compare the overall axonal transport and presynaptic targeting of synapsin, one of the best-studied SCb proteins (Baitinger and Willard, 1987; Petrucci et al., 1991; Paggi and Petrucci, 1992), with synaptophysin, a trans-membrane protein largely moving in fast axonal transport (Elluru et al., 1995), using a imaging paradigm that allowed us to compare the transport behavior of these two proteins over long time scales.

Simulating the paradigm used in pulse-chase radiolabeling experiments, we visualized the emergence of newly synthesized cytosolic proteins from the soma into axons. Specifically, we transfected cultured hippocampal neurons with GFP:synapsin (and soluble mCherry for axon identification), and then visualized the entry of GFP-fluorescence into axons or boutons at early time points $(4-5 \mathrm{~h}$ ) after transfection (see strategy in Figure $1 A$; Materials and Methods). We reasoned that these time points, close to known maturation times of $\mathrm{GFP} / \mathrm{mCherry}$ chromophores, would represent the earliest times at which these proteins would be expressed in our neurons. Figure $1 B$ is a representative example of one such experiment, showing the entry of newly synthesized synapsin into an axon devoid of fluorescence at $t=0$. There is slow ingress of labeled synapsin molecules over several hours of imaging (Fig. 1C, left, kymographs). More rapid image acquisition highlights the emergent "wave-like" motility of synapsin molecules (Fig. 1C, right). The slow overall dynamics of synapsin is very different from the fast axonal transport of synaptophysin, where a deluge of rapidly moving vesicles enter axons (Fig. 1D) (Scott et al., 2011; their Fig. 5). 
A Concept of quantitative algorithm
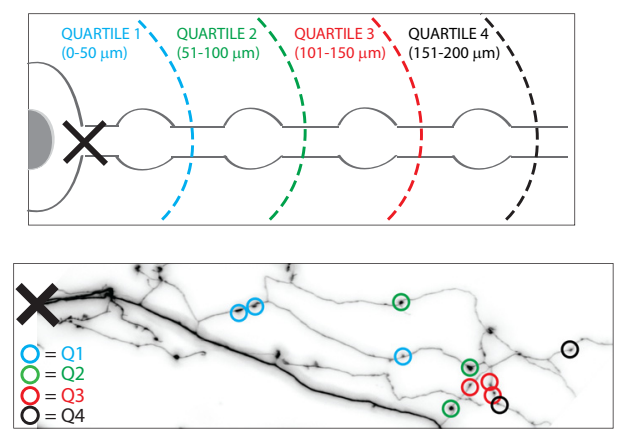

C GFP:synapsin accumulation at boutons
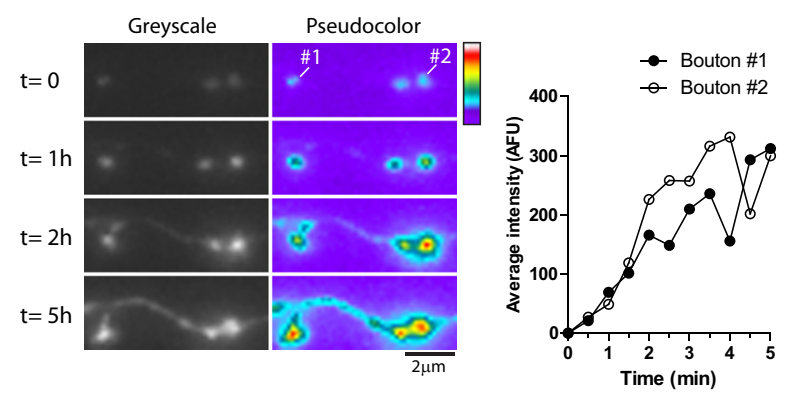

B GFP:Synapsin: presynaptic targeting

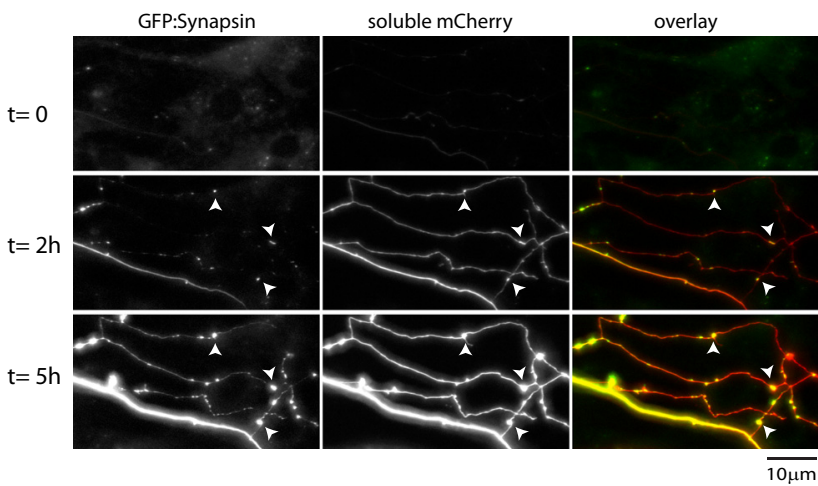

D

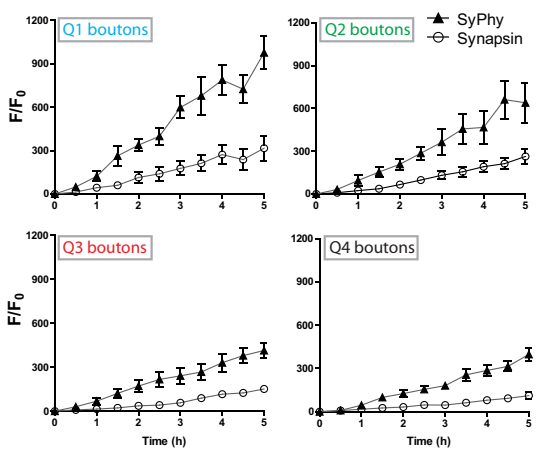

\section{E Comparison of synaptophysin and synapsin data from all quartiles}
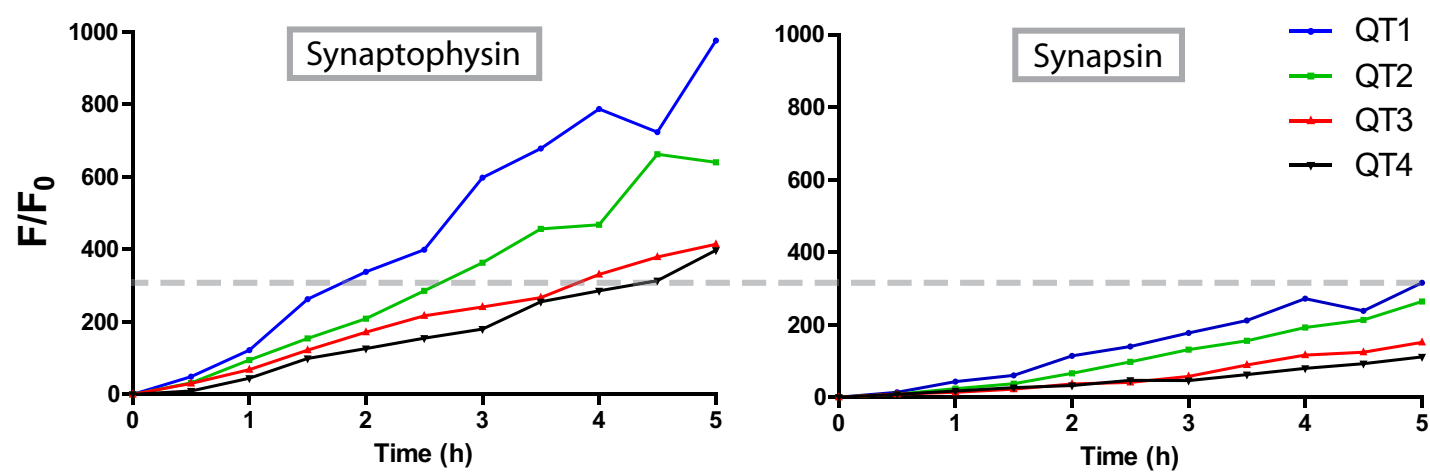

Figure 2. Characterizing overall kinetics of slow and fast transport proteins in cultured hippocampal neurons. $A$, A method to quantify accumulation of slow and fast component proteins (synapsin and synaptophysin respectively) in boutons. Neurons were transfected/imaged as described in Figure $1 A$, and fluorescence accumulation within boutons was quantified over $5 \mathrm{~h}$ of imaging. Quantitative algorithms were developed to pinpoint the distance of a given bouton from the soma/axon junction (indicated by $\times$ ). For quantification, boutons from all axons analyzed were binned into quartiles (Q1-Q4), $50 \mu \mathrm{m}$ bins based on their distance from the soma, and fluorescence accumulation in boutons within each bin was analyzed as a group. $\boldsymbol{B}$, Selected panels on left, The axonal accumulation of GFP:synapsin and a corresponding accumulation in distal boutons over a period of $5 \mathrm{~h}$ ( $t=0$ denotes the first time point when the axons were imaged). In contrast, soluble $\mathrm{mCherry}$ is rapidly distributed throughout the axons, as diffusion is rapid at these short distances (middle panels). Right panels, Overlay of the two proteins: green represents synapsin; red represents mCherry. C, Left panels, An example of GFP:synapsin accumulation in boutons over $5 \mathrm{~h}$; average intensities of two boutons (1 and 2) quantified on right. D, Quantification of average intensities in boutons over $5 \mathrm{~h}$ (normalized to initial intensities within each bouton, F0). Although both synaptophysin (triangles) and synapsin (circles) intensities incrementally rise with time, the kinetics of synapsin accumulation was slower than synaptophysin for all quartiles examined. $\boldsymbol{E}$, Side-by-side comparison of all synaptic accumulation data; $\sim 50$ boutons from three separate culture sets were examined for each protein. There are slower kinetics of synapsin accumulation for all boutons examined.

As both synapsin and synaptophysin localize to presynaptic boutons (Fletcher et al., 1991), another way to evaluate their overall motility is to quantify protein accumulation at boutons over time. Toward this, we developed quantitative algorithms that pinpointed the distance of any given bouton from the soma (see concept in Fig. 2A; Materials and Methods). As branching patterns in cultured hippocampal neurons are typically complex and any given bouton ROI location depends on the somatodendritic architecture of the neuron in question (where the boutons are typically established), quantitative analysis was done on groups of boutons that were binned based on their distance from the soma. Specifically, boutons that were within $200 \mu \mathrm{m}$ from the soma were divided into four (equal) quartiles, and all boutons within each quartile were binned and evaluated as a group (Fig. $2 A)$. This allowed us to reliably compare data from different neurons and perform robust quantification.

A typical example from one such experiment is shown in Figure $2 B$, where boutons were imaged for up to $5 \mathrm{~h}$. There is a gradual increase in GFP:synapsin fluorescence in boutons over time (Fig. 2C). Quantification of synapsin and synaptophysin 


\section{A Strategy to evaluate effects of vesicle-transport inhibition on slow axonal transport}

\begin{tabular}{|c|c|c|c|c|}
\hline $\begin{array}{l}\text { Co-transfect neurons } \\
\text { with PAGFP-tagged SCb } \\
\text { protein + sol mRFP }\end{array}$ & $\begin{array}{l}\text { INHIBIT } \\
\text { VESICLE- } \\
\text { TRANSPORT } \\
\text { (see B.) }\end{array}$ & $\begin{array}{l}\text { Select primary } \\
\text { axon using RFP }\end{array}$ & $\begin{array}{l}\text { Photoactivate } \\
\text { PAGFP:Synapsin }\end{array}$ & $\begin{array}{l}\text { Evaluate fluorescence } \\
\text { kinetics using 'intensity } \\
\text { center shift' assay } \\
\text { (see text) }\end{array}$ \\
\hline
\end{tabular}

\section{B Protocols to inhibit vesicle-transport}

\begin{tabular}{|c|c|c|c|c|c|c|c|}
\hline $\begin{array}{l}\text { (1) Transfect } \\
\begin{array}{l}\text { tagged SyPhy } \\
\text { (or Mito) }\end{array}\end{array}$ & Add BFA & {$\left[\begin{array}{c}\text { Image } \\
\text { axonal } \\
\text { transport }\end{array}\right.$} & $\begin{array}{l}\text { (2) Co-transfect } \\
\begin{array}{l}\text { tagged SyPhy } \\
\text { (or Mito) } \\
+ \text { ARF-1 T31N }\end{array}\end{array}$ & $\begin{array}{c}\text { Image } \\
\text { axonal } \\
\text { transport }\end{array}$ & $\begin{array}{l}\text { (3) Transfect } \\
\text { tagged SyPhy } \\
\text { (or Mito) }\end{array}$ & {$\left[\begin{array}{l}19^{\circ} \mathrm{C} \\
\text { Temp } \\
\text { block }\end{array}\right.$} & {$\left[\begin{array}{c}\text { Image } \\
\text { axonal } \\
\text { transport }\end{array}\right.$} \\
\hline ; 16-20h & $\overline{3 h}$ & & 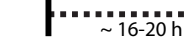 & & $\sim 16-20 \mathrm{~h}$ & $2 \mathrm{~h}$ & \\
\hline
\end{tabular}

C SyPhy kymos
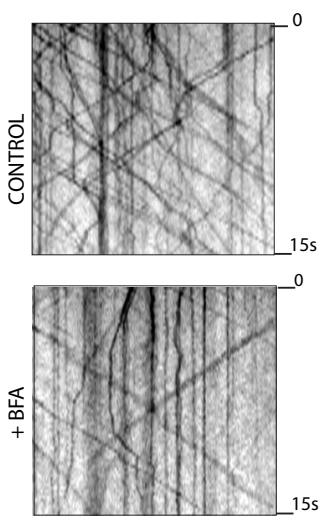

D
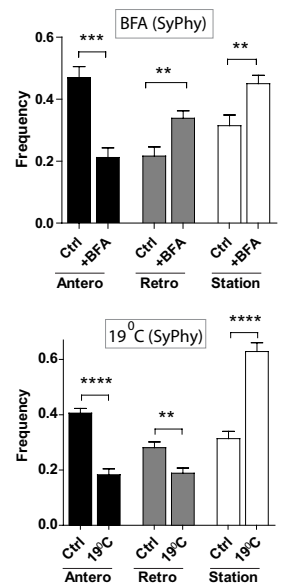

E
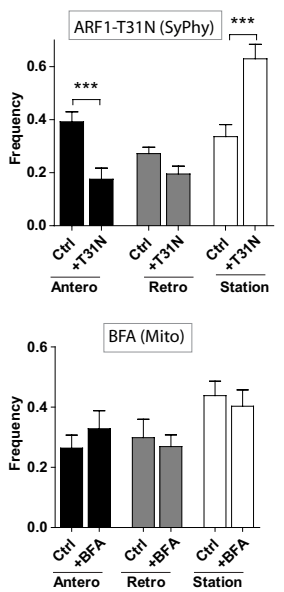

E Synapsin targeting to synapses
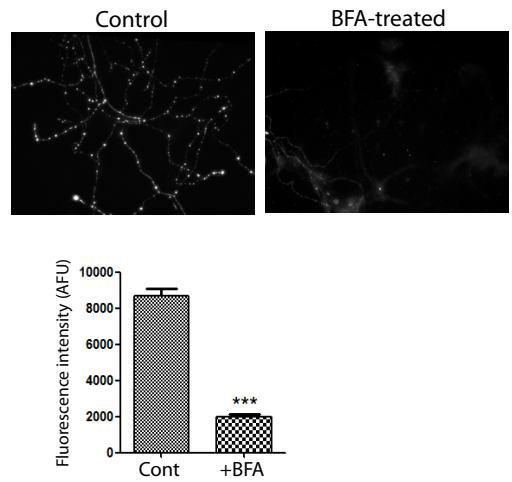

Figure 3. Inhibiting axonal transport of Golgi-derived vesicles in cultured neurons. $\boldsymbol{A}$, Photoactivation strategy to evaluate slow axonal transport of synapsin (region between arrows was photoactivated). The anterograde bias of photoactivated synapsin was used as a readout of its slow axonal transport (see Materials and Methods). $\boldsymbol{B}$, Neurons underwent various manipulations to disrupt export of Golgi-derived vesicles (also see Results), and axonal transport of synaptophysin (SyPhy):mRFP or Mito:mRFP was evaluated. C, Examples of SyPhy:mRFP kymographs from control/BFA-treated axons highlight diminutions in vesicle transport. Axons were imaged at 5 frames/s; time elapsed is on the right of the kymographs. D, Quantification of all transport data indicates that the above manipulations also decreased anterograde transport of synaptophysin and led to increases in stalled particles. However, BFA treatments did not alter axonal transport of mitochondria, suggesting specific diminutions of Golgi-derived vesicles; $\sim 10-20$ axons (200-600 particles) from two separate culture sets were analyzed in each condition. $\boldsymbol{E}$, Synaptic targeting of GFP-synapsin was also greatly diminished after BFA treatment, as shown in the representative images on top (normalized to the same range) and quantitation of "targeting factor" below (see Results).

accumulation in boutons, normalized to the initial fluorescence intensities $\left(\mathrm{F}_{0}\right)$ in boutons, is shown in Figure $2 D$. The rate of synaptophysin accumulation is significantly faster than synapsin in all binned datasets. A side-by-side comparison of the cumulative data from all synaptophysin and synapsin imaging experiments is shown in Figure 2E. Collectively, these experiments establish that the bulk movement of the SCb protein synapsin is slower than synaptophysin in our model system, and the overall population kinetics are very different (movement of a fluorescence "wave" vs stochastic transport of individual vesicles). Moreover, the design of these experiments should mitigate potential concerns related to overexpression that are unavoidable in live-cell transport studies.

\section{Fast vesicle transport is required for slow axonal transport} of synapsin

The above experiments show that the overall kinetics of synapsin and synaptophysin are very different in cultured neurons. What is the underlying basis for the biased flow of cytosolic molecules in axons? One possibility is that synapsin molecules assemble into "transport-competent" complexes that subsequently associate with vesicles that are persistently moving in fast axonal transport. In support of this idea, we have noticed persistent linear "tracks" in synapsin kymographs (Tang et al., 2012a), suggesting that synapsin molecules were associating with rapidly moving organelles resembling vesicles. Accordingly, we asked whether slow axonal transport of synapsin was dependent upon vesicle transport.

The general strategy of these experiments is shown in Figure $3 A$. Essentially, slow axonal transport of synapsin was evaluated in neurons where vesicle transport was experimentally inhibited. To visualize and quantify slow axonal transport, we used our previously reported assays (Scott et al., 2011; for detailed methods, see Roy et al., 2011). In this assay, neurons are transfected with the PAGFP-tagged SCb protein of interest (and soluble mRFP to identify transfected axons). Thereafter, a discrete ROI within the primary axon is photoactivated, and the dispersion of activated fluorescent molecules in axons is monitored by timelapse imaging. The photoactivated SCb population disperses with an anterogradely biased flow that superficially resembles diffusion but is microtubule/motor/ATP-dependent (Scott et al., 
A

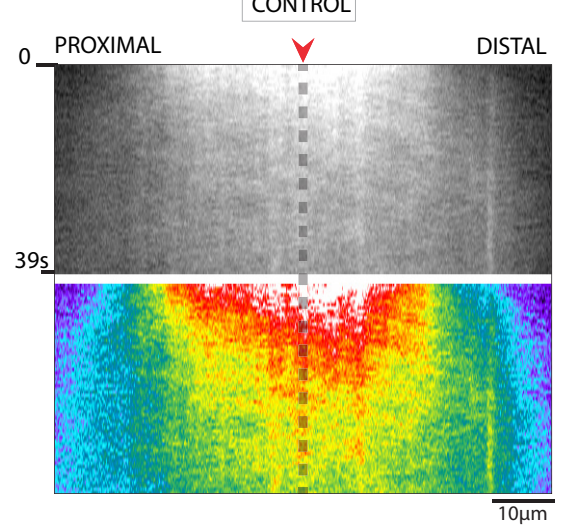

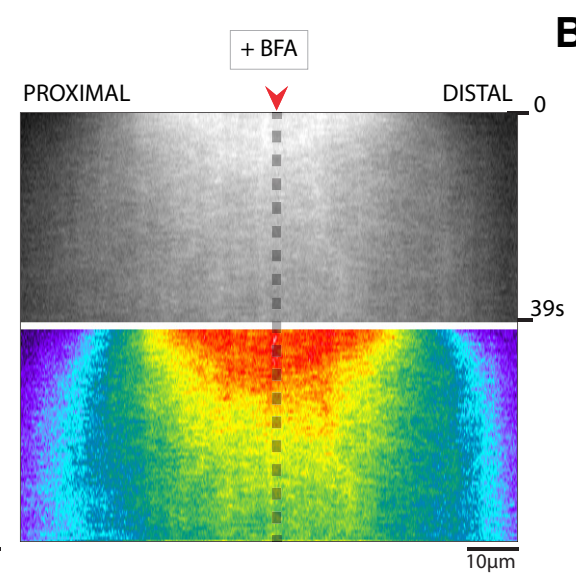

B Raw shifts

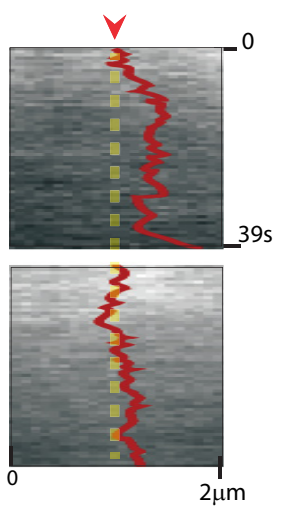

\section{Synapsin transport}

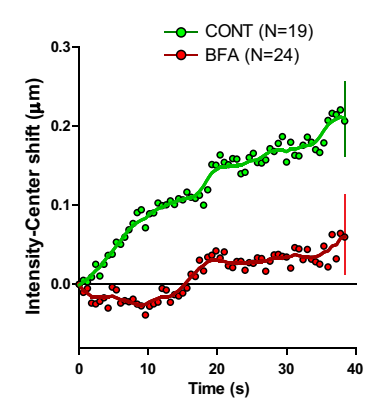

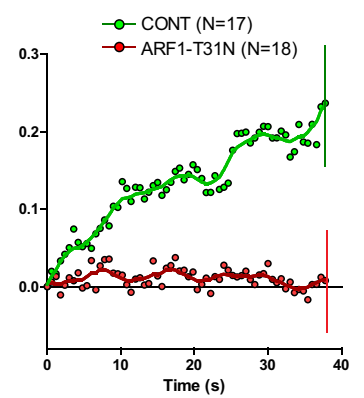

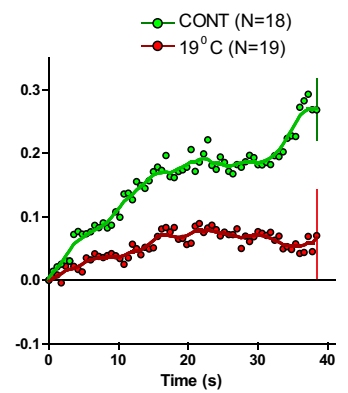

\section{Untagged PAGFP}

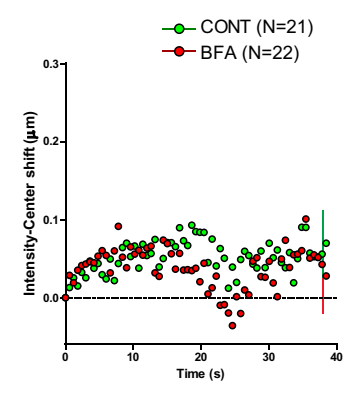

Figure 4. Slow cytosolic transport is inhibited by disruptions in vesicle transport. $\boldsymbol{A}$, Kymographs show dispersion of photoactivated PAGFP:synapsin in axons. Red arrowhead and dashed vertical line indicate the center of the photoactivated zone. In control axons (left panels), PAGFP disperses as a plume of fluorescence with an anterograde bias (left to right is proximal to distal), whereas this bias is attenuated in BFA-treated axons (right panels). $\boldsymbol{B}$, Raw "intensity-center shifts" of the kymographs from $\boldsymbol{B}$, depicting the anterograde displacement/slow transport of the synapsin population (see Results). C, Mean intensity-center shifts and curve-fits for all experiments with PAGFP:synapsin. There is diminution of synapsin transport by BFA, ARF-1, and $19^{\circ} \mathrm{C}$ incubation. $\boldsymbol{D}$, As expected for free diffusion, there was no sustained bias of untagged PAGFP in axons, with or without BFA. $N=$ number of axons imaged; $2-4$ separate culture sets were imaged for each condition.

2011) (e.g., see Fig. 4A). The rate of this biased flow is quantified by custom algorithms that calculate the overall shift in the centroid of the photoactivated population at each time point ('intensity-center shift'), and the data are plotted as 'intensitycenter' shift/time (Fig. 4C). Bulk movement of SCb molecules give rise to a slow, anterograde bias of photoactivated molecules that is similar to the rates predicted by radiolabeling studies (see Materials and Methods; Roy et al., 2011).

Three strategies were used to inhibit vesicle transport (Fig. 3B): (1) Neurons were treated with BFA, a pharmacologic agent that disrupts the Golgi complex. (2) Neurons were transfected with ARF1-T31N, a constitutively active version of the GTPase ARF-1 that blocks vesicles from exiting the Golgi (Dascher and Balch, 1994). (3) Neurons underwent a transient temperature block $\left(19^{\circ} \mathrm{C}\right)$, a relatively noninvasive way of perturbing ER $\rightarrow$ Golgi transport (Dresbach et al., 2006). As shown in Figure 3C, D and Table 1, these manipulations led to a decrease in the relative numbers of anterogradely moving vesicles with corresponding increases in stationary pools, indicating that fast vesicular transport was indeed disrupted by these procedures. Importantly, axonal transport of mitochondria, the biogenesis of which is thought to be independent of the ER $\rightarrow$ Golgi, was not significantly affected by BFA, suggesting that these interventions were not creating global blocks in axonal transport. Expectedly, BFA treatment also dramatically diminished the targeting of synapsin to synapses (Fig. 3E).

Next, we evaluated effects of the above manipulations on the slow axonal transport of synapsin. Figure $4 A, B$ shows represen-
Table 1. Frequency (relative to total number of particles) and velocities (average $\mu \mathrm{m} / \mathrm{s}$ )

\begin{tabular}{llll}
\hline & Anterograde & Retrograde & Stationary \\
\hline $\begin{array}{l}\text { Frequency (relative to total number of particles) } \\
\text { BFA treatment }\end{array}$ & & & \\
$\quad$ Control & 0.47 & 0.22 & 0.31 \\
$\quad$ BFA & $0.21^{* *}$ & $0.34^{*}$ & $0.45^{*}$ \\
ARF-1 inhibition & & & \\
$\quad$ Control & 0.39 & 0.27 & 0.34 \\
$\quad$ T31N & $0.17^{* *}$ & 0.19 & $0.63^{* *}$ \\
Temporary block & & & \\
$\quad$ Control & 0.41 & 0.28 & 0.31 \\
19 & $0.18^{* * *}$ & $0.19^{*}$ & $0.63^{* * *}$ \\
Velocities (average $\mu \mathrm{m} / \mathrm{s})$ & & & \\
BFA treatment & & & \\
$\quad$ Control & 1.78 & 1.94 & - \\
$\quad$ BFA & 1.77 & $1.46^{* *}$ & - \\
ARF-1 inhibition & & & - \\
$\quad$ Control \\
$\quad$ T31N \\
Temporary block \\
$\quad$ Control \\
$\quad 19^{\circ} \mathrm{C}$
\end{tabular}

${ }^{*} p<0.001 ;{ }^{* *} p<0.0001 ;{ }^{* * *} p<0.00001$.

tative kymographs/raw intensity-center shifts from one such experiment with BFA. The anterogradely biased plume of fluorescence (better appreciated in pseudocolor images) is attenuated in BFA-treated axons. Figure $4 C$ shows the mean intensity-center shifts and corresponding curve-fits for all axons examined in 
control and experimental groups where vesicle transport was attenuated. In all cases, experimental treatments that inhibited fast vesicle transport also attenuated the slow axonal transport of synapsin. The dispersion of untagged PAGFP was unbiased as described previously (Scott et al., 2011), and addition of BFA did not have any perceptible influence on these kinetics (Fig. 4D).

\section{Association with vesicles is required for the slow axonal transport of synapsin}

We previously reported that, although the net intensity-center shift of synapsin in a given dataset, as determined by imaging several axons, is consistently anterograde, there is inherent variability from axon to axon (Roy et al., 2011). For example, the individual fluctuations in Figure $5 A$ (top) show all the raw intensity-center shifts obtained from individual axons for the control synapsin dataset shown in Figure 4C. Similar fluctuations in intensity-center shifts were seen, even when the same axon was repeatedly photoactivated (Fig. $5 A$, bottom), indicating that this is a biologic variation and not a technical issue. Similar fluctuations in vesicle motility kinetics are also apparent in fast axonal transport (Fig. 5B-D), also reported previously (Zahn et al., 2004; Tang et al., 2012b). Although the exact reasons for these variations are unclear, we reasoned that, if synapsin transport is indeed dependent upon fast axonal transport of vesicles, intrinsic fasttransport variations in a given axon should also lead to analogous changes in slow axonal transport in the same axon.

To test this idea, we cotransfected neurons with synaptophysin:mRFP and PAGFP:synapsin, sequentially imaged both fast and slow axonal transport in the same axon (Fig. 6A, "tandemimaging strategy"), and correlated synaptophysin and synapsin transport parameters in the same axon. Two contrasting examples of synaptophysin transport are shown in Figure 6A (axons 1 and 2, left and right panels). There are analogous changes in intensity-center shifts of synapsin (middle panels). Although there are transport fluctuations within the same axon over time (as noted previously), these are not apparent during the brief imaging periods (60s) used in these experiments, and frequencies of moving synaptophysin particles were similar in the "before" and "after" groups (Fig. 6B). Figure 6C, D shows cumulative quantitative data from all such experiments. Although average synapsin shifts in axons correlated with the number of mobile anterograde synaptophysin particles, such correlations were not seen with retrogradely moving or stationary particles. These data suggest that synapsin assemblies preferentially associate with anterogradely moving vesicles. Indeed, previous studies using the "stop flow" (or crush) technique in rat peripheral nerves have shown almost exclusive accumulation of synapsin proximal to the crush lesions, with little or no accumulation distally (for review, see Dahlström et al., 1992; and see Discussion).

Synapsin is known to physically associate with vesicles; however, unlike trans-membrane proteins, these associations are transient and regulated by a number of factors, including phosphorylation and intracellular calcium levels (for review, see Cesca et al., 2010). Is the physical association of synapsin with vesicles a requirement for its slow axonal transport? To test this, we first asked whether synapsin fragments that fail to associate with vesicles also show attenuated anterograde bias in our photoactivation assay. Synapsin has various domains, known as "A" to "F." It is known that the $\mathrm{C}$ domain mainly associates with vesicles and that such associations also involve the $\mathrm{A}$ and $\mathrm{B}$ domains, but the DE fragment does not play any known role in vesicle binding (Cheetham et al., 2001, 2003). Thus, we evaluated the axonal transport of the DE fragment using PAGFP:DE-synapsin. As shown by Gitler et al. (2004), the synaptic targeting of the DE fragment was much lower than the WT protein (Fig. 6E, left, inset). Interestingly, the anterograde bias of PAGFP:DE-synapsin was greatly attenuated compared with the WT protein (Fig. $6 E$, left). Next, raising intracellular calcium levels by ionomycin, known to displace synapsin from vesicles (Denker et al., 2011) also attenuated the slow axonal transport of synapsin (Fig. 6E, middle). Finally, we used pharmacologic agents to modulate the phosphorylation status of synapsin in our neurons. Specifically, protein kinase A (PKA) phosphorylation is known to decrease membrane binding of synapsin (Cesca et al., 2010), and the PKA activator forskolin has been shown to detach synapsin from synaptic vesicles (Menegon et al., 2006). If our hypothesis is correct, one would expect that forskolin treatment would abolish association of synapsin molecules with anterogradely moving vesicles and attenuate its slow axonal transport. As shown in Figure $6 E$ (right), forskolin treatment actually induced a retrograde bias to the movement. In contrast, treatment of neurons with the PKA inhibitor H89 had little effect on synapsin transport. Although the reason behind the retrograde bias is unclear, phosphorylation events may differentially regulate the association of synapsin with anterograde/retrograde vesicles. Nevertheless, these data suggest that the physical association of synapsin with vesicles is an important determinant of its slow axonal transport.

\section{A conceptual framework using long-term simulations: dynamic associations of soluble assemblies with vesicles}

Previous radiolabeling studies have shown that, although the bulk of synapsin is conveyed in SCb, small amounts ( $\sim 15 \%$ of the label) move with much faster kinetics that resembles fast axonal transport (Baitinger and Willard, 1987; Paggi and Petrucci, 1992). Similar phenomena have been reported for other cytosolic proteins moving in slow transport as well (Black et al., 1991; Jensen et al., 1999; Susalka et al., 2000). In previous experiments, we saw that photoactivation of PAGFP:synapsin in cell bodies resulted in a slowly emerging "wave" in axons (as expected for $\mathrm{SCb}$ proteins), but rapid anterogradely biased particles were also seen distal to the "wave-front" (Scott et al., 2011, their Fig. 4A), suggesting that, like the in vivo situation, a fraction of SCb was also being conveyed in fast axonal transport. How is this faster movement generated? One possibility is that, immediately after synthesis in the soma, a subpopulation of cytosolic particles, with perhaps distinct biochemical properties, is continuously associated with vesicles and exported rapidly. However, if we consider that synapsin assemblies probabilistically bind to vesicles with a range of interaction strengths, another possibility is that the faster population simply represents a fraction that happens to remain associated with vesicles for longer times.

As technical limitations do not allow us to simultaneously monitor the somatic export of SCb cargoes as well as their mobility in axons hundreds of microns away, we used adaptations of our previous biophysical simulations (Scott et al., 2011) to address this. Briefly, the simulation assumes an aqueous cylindrical environment (axon) containing two main components: (1) cytosolic/SCb particles that are free to diffuse (with relevant diffusion constants; see Materials and Methods) and/or collide with other cytosolic particles; and (2) rapid and persistent "mobile units" (hereafter called "vesicles") that traverse along the axon with a range of fast transport velocities (obtained from our synaptophysin imaging datasets, $1.8 \pm 0.7 \mu \mathrm{m} / \mathrm{s}$, mean $\pm \mathrm{SD})$. A critical component of this simulation is that, in addition to random collisions, the cytosolic particles are allowed to interact with the moving 
A Fluctuations in slow transport B Fluctuations in fast transport
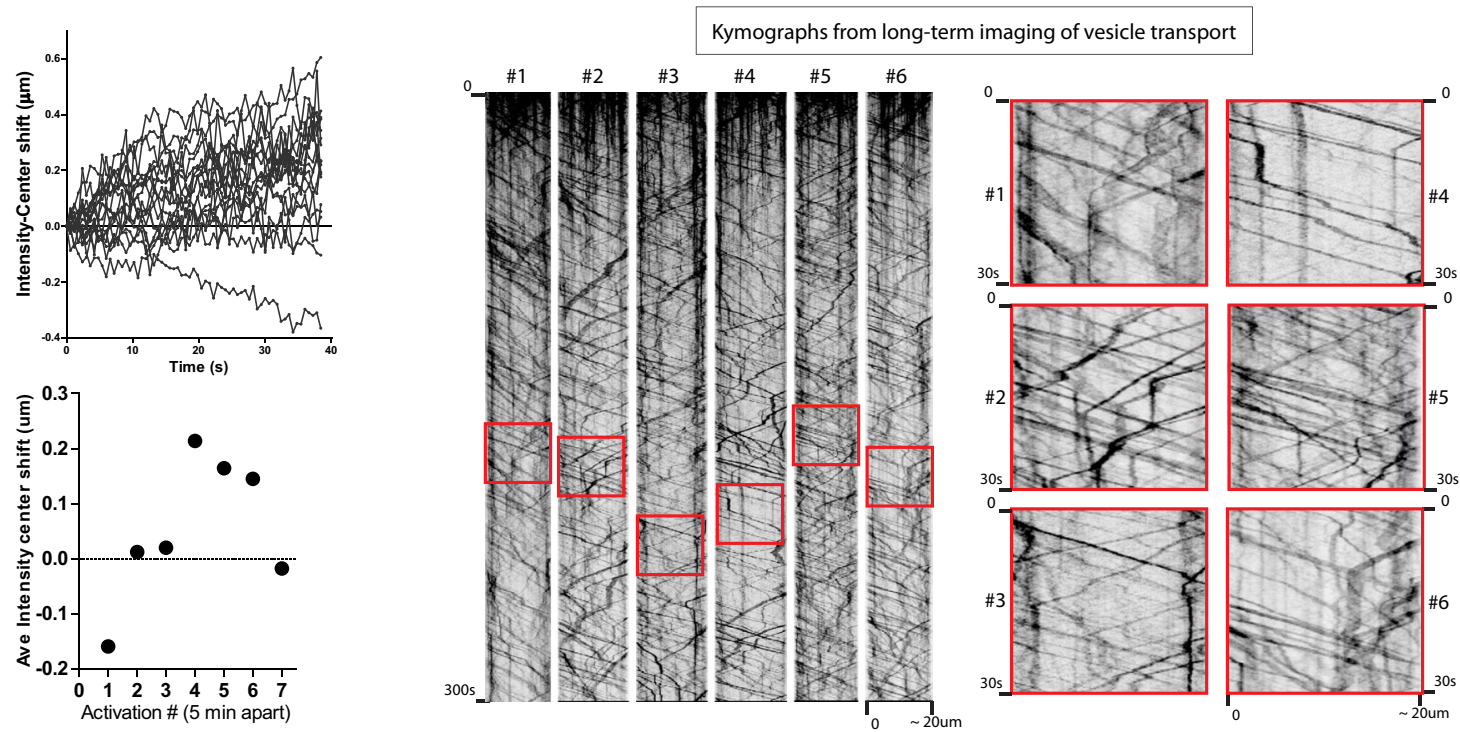

\section{Long-term imaging of vesicle-transport}
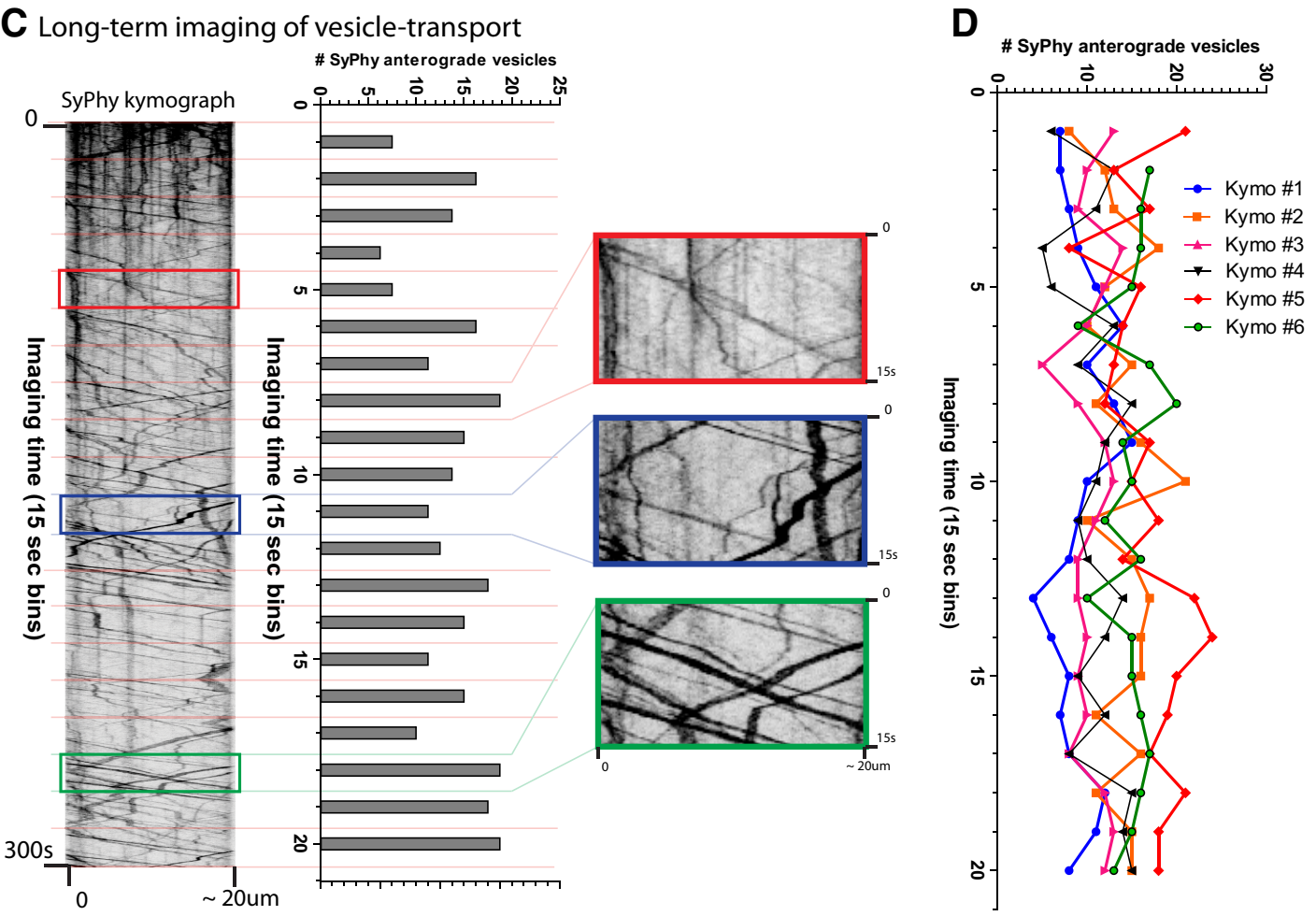

Figure 5. Intrinsic fluctuations in slow and fast axonal transport. A, Top, Individual PAGFP:synapsin intensity-center shifts of all axons examined for a single dataset (Fig. 4D, left, from the "control" group). There is variability in individual shifts. Bottom, To evaluate potential intrinsic fluctuations in synapsin transport within the same axon, PAGFP:synapsin was repeatedly photoactivated (at 5 min intervals) and time-lapse imaging was performed to determine intensity-center shifts at each time point. Each data point in the graph represents the average shift from a single time-lapse movie ( > 40 s of imaging time). There is variability in synapsin transport within the same axon over time. B, Synaptophysin:mRFP transport was visualized at 5 frames $/ \mathrm{s}$ for $300 \mathrm{~s}$ of total imaging time using protocols that tracked all (or most) moving particles with minimal bleaching (see Materials and Methods); 30 s cropped insets on right (red boxes) highlight variations in transport frequency. $\boldsymbol{C}$, Each kymograph was divided into $15 \mathrm{~s}$ bins (horizontal red lines in kymograph shown), and the number of mobile anterograde particles in each bin was quantified. Three such bins are zoomed on right. There are intrinsic fluctuations in synaptophysin transport. D, Cumulative data from several axons also highlight such transport variations from axon to axon. SyPhy, Synaptophysin.

vesicles with user-defined interaction strengths. Virtual kymographs and resultant intensity-center shifts are generated from these simulations (for further details, see Materials and Methods).

To simulate emergence of cytosolic particles from the soma into axons, we placed the cytosolic particles on one end of the cylinder (axon). A long (175 $\mu \mathrm{m})$ cylindrical axon, devoid of any cytosolic particles at $t=0$, was considered, so that the entire gamut of SCb motility kinetics could be appreciated (Fig. 7A, virtual kymographs). A physical interaction of the cytosolic particles with vesicles is required for sustained transport, and diffusion alone is unable to generate robust shifts (Fig. $7 B-D$ ). Cumulative data from several simulations show that the average 
A
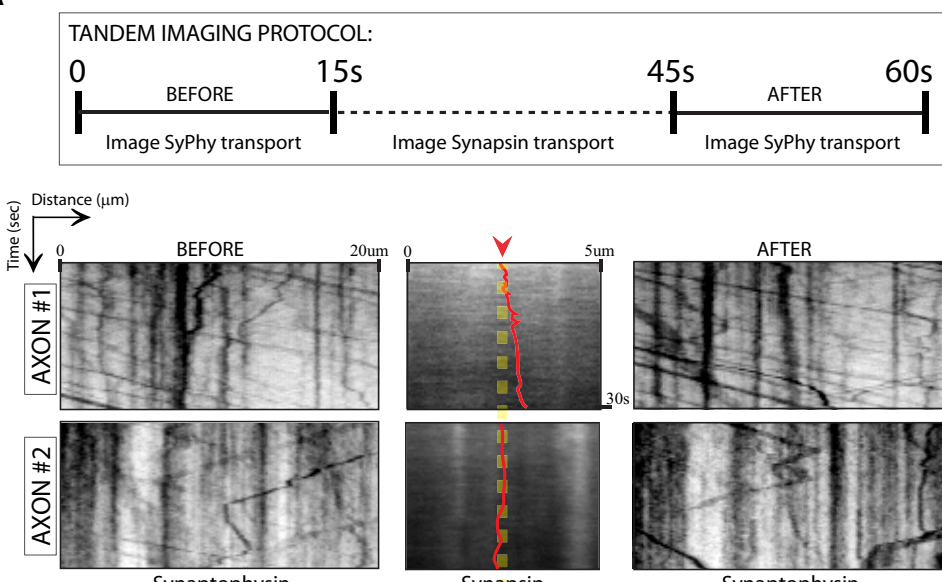

Synaptophysin
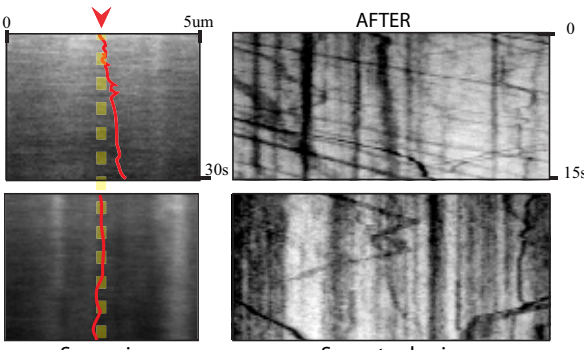

Synapsin

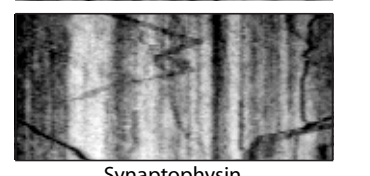

C

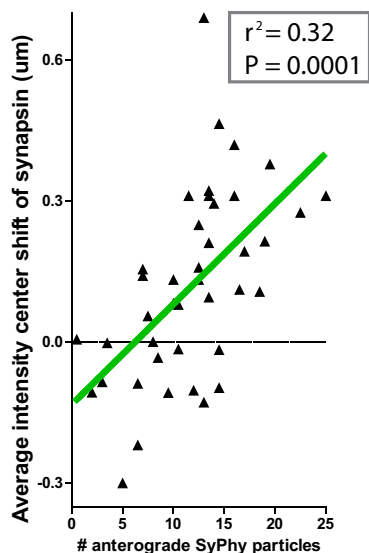

B

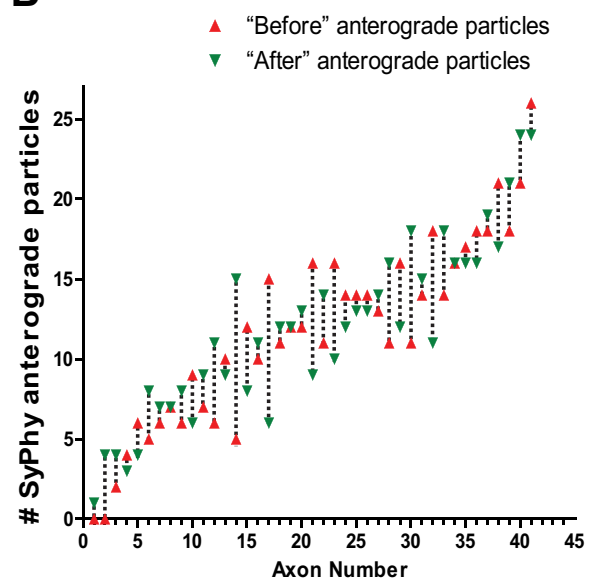

D

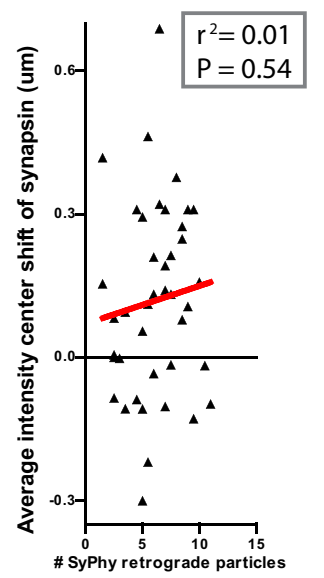

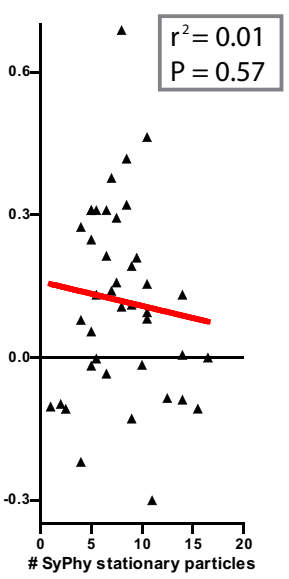

E Association with vesicles is required for synapsin transport
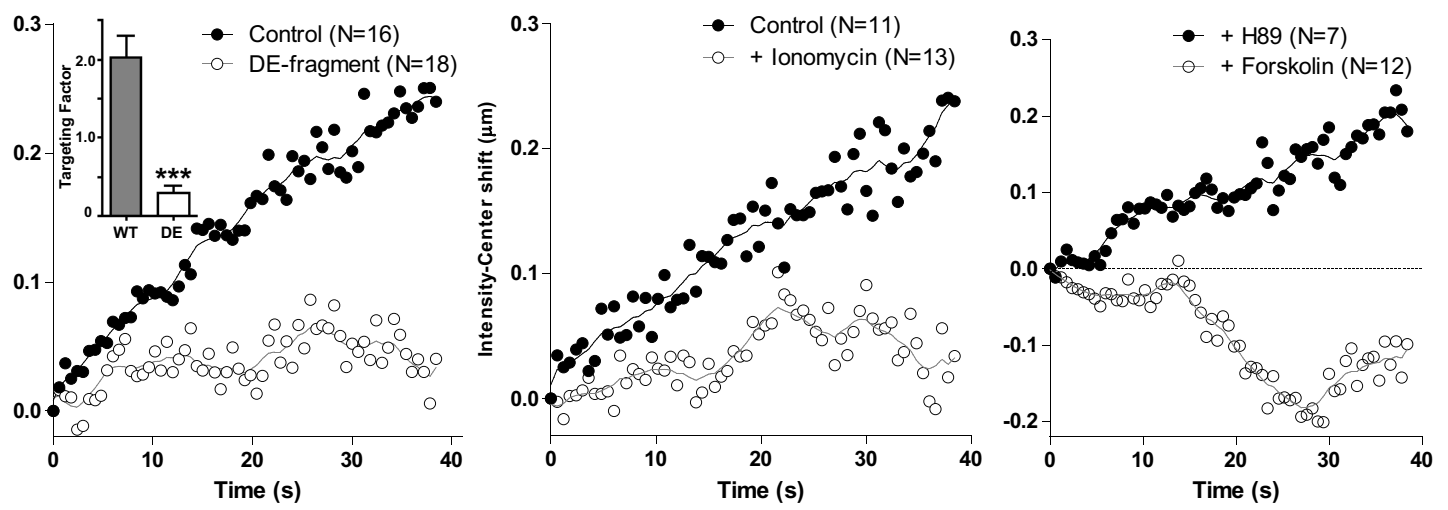

Figure 6. Correlated fluctuations of fast and slow axonal transport. A, Tandem imaging protocol. Neurons were transfected with synaptophysin (SyPhy:mRFP) and PAGFP:synapsin, and axonal transport of both synaptophysin and synapsin was sequentially analyzed in the same axon as described in the text. Bottom, Kymographs of SyPhy and synapsin imaging from two axons. There is robust SyPhy and synapsin transport (the latter determined by intensity-center shifts) in axon 1 but diminished transport of both in axon 2 . B, The number of mobile anterograde SyPhy particles were not altered in the "before" and "after" datasets, indicating that transport parameters within a given axon were generally similar during the short ( 60 s) of total imaging. C, Average intensity-center shifts of synapsin in axons significantly correlated with the number of motile anterograde SyPhy particles; however, no correlations were seen with retrograde/stationary particles $(\boldsymbol{D})$. Approximately 40 axons from 5 separate culture sets were analyzed. $\boldsymbol{E}$, Left, Using the photoactivation assay, slow axonal transport of a PAGFP-tagged synapsin fragment (PAGFP:DE) was compared with the full-length protein. This synapsin fragment lacks the domains that are involved in association of synapsin with vesicles (see Results). Anterograde bias of the DE domain was greatly attenuated. Presynaptic targeting of a GFP:DE construct was also greatly diminished (inset), confirming its weak association with vesicles (synaptic "targeting factor" quantified as in Gitler et al., 2004). Middle, Incubation of neurons with ionomycin (10 $\mu \mathrm{m}$ for $30 \mathrm{~min}$ ), expected to dissociate synapsins from vesicles, also inhibited synapsin transport. Right, PKA activation by forskolin ( $50 \mu \mathrm{m}$ for $30 \mathrm{~min}$ ) induced a retrograde bias in synapsin transport, but the PKA inhibitor H89 (10 $\mu \mathrm{m}$ for $30 \mathrm{~min}$ ) did not influence the anterograde bias of synapsin (see Results). 
A

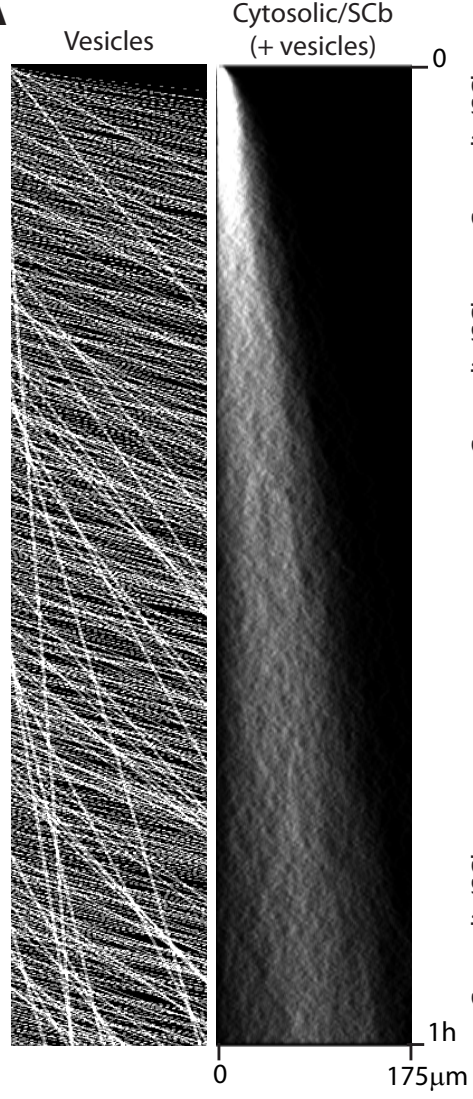

B
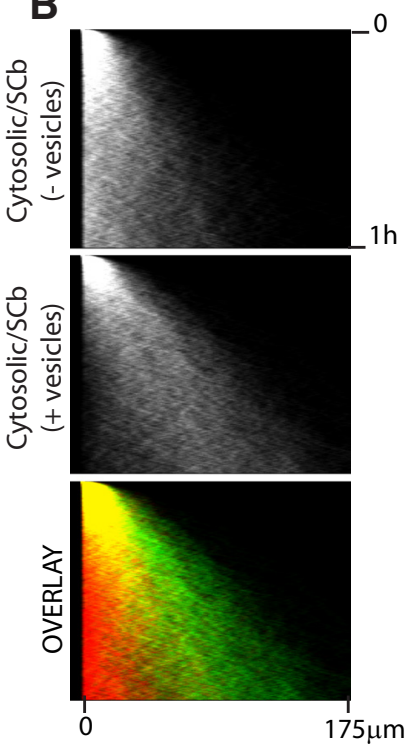

C

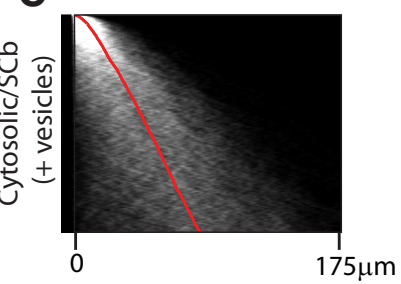

D
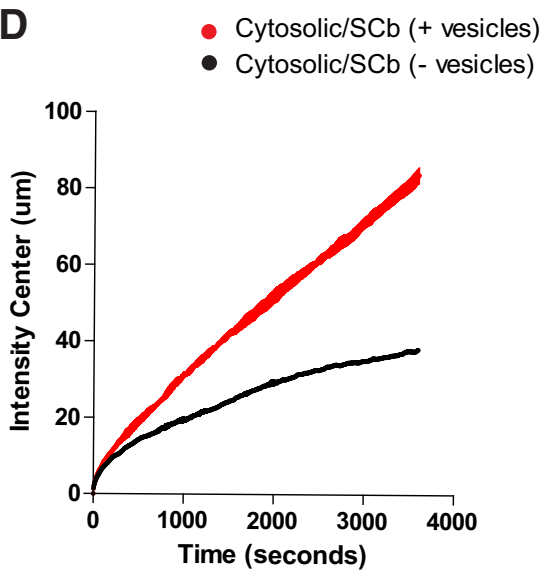

E Scaled simulated kymographs

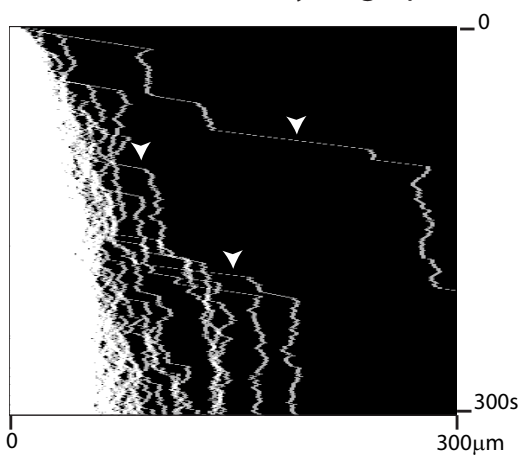

F Modeling vesicle-associations with $\mathrm{SCb}$ cargoes
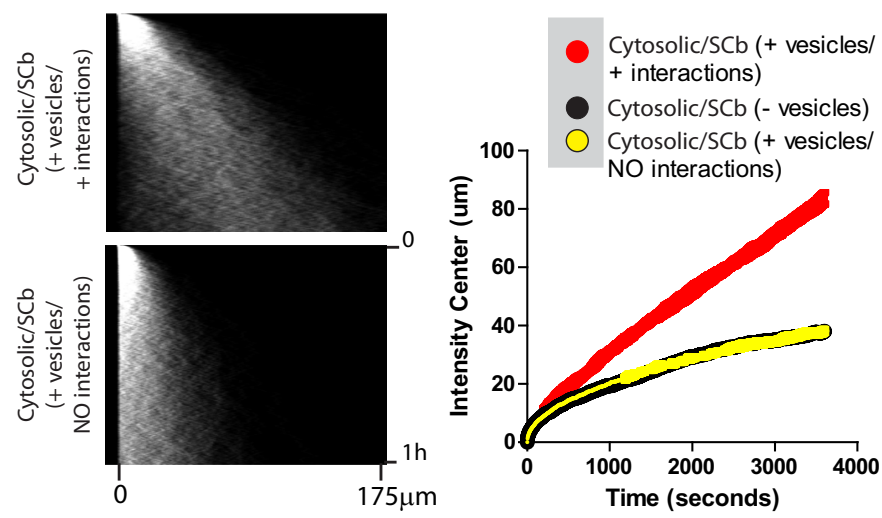

Figure 7. Simulations of fast vesicular and cytosolic slow axonal transport. $A, A$ virtual environment was created to simulate motile vesicles (left kymograph) and cytosolic/SCb particles (right kymograph) emerging from a hypothetical "cell body" into an "axon" (anterograde is left to right, time on $y$-axis). In addition to diffusion, cytosolic particles were allowed to interact with vesicles for user-defined times. Using these simulations, particle motility can be modeled over long distances and for prolonged periods of time, unattainable by conventional imaging. $\boldsymbol{B}$, Simulated kymographs of cytosolic particles into axons with and without concurrent ingress of motile vesicles. The movement of cytosolic particles is greatly facilitated by the presence of motile vesicles. $C$, Intensity-center shifts (red line) were generated from the virtual kymographs (see Materials and Methods). $\boldsymbol{D}$, Intensity-center shifts of the cytosolic/SCb population (mean \pm SD shifts from four independent simulations are shown). The predicted average transport rate of the cytosolic population from these simulations was $0.02109 \mu \mathrm{m} / \mathrm{s}(\sim 2 \mathrm{~mm} / \mathrm{d})$, in line with $\mathrm{known} \mathrm{SCb}$ rates from radiolabeling studies. E, When kymographs were scaled to reveal all moving particles, occasional persistent particles, distal to the slowly progressing "wave," were also seen (see Results). $\boldsymbol{F}$, Sustained anterograde shifts in simulations (red data points) were only seen when interactions between vesicles/SCb cargoes were assumed, and movement of vesicles alone (without vesicle/ $\mathrm{SCb}$ cargo interactions) were insufficient to generate robust transport (yellow data points).

velocity $(\sim 2 \mathrm{~mm} / \mathrm{d})$ of the migrating "wave-front" (Fig. $7 D)$ is well in line with expected $\mathrm{SCb}$ rates (Baitinger and Willard, 1987). Next, we focused on individual particle kinetics in these simulations, maximizing the bit depths in virtual kymographs to reveal motility of single synapsin particles distal to the wavefront. Persistently mobile particles were clearly seen in such simulations, hundreds of microns distal to the slowly moving $\mathrm{SCb}$ "wave-front" (Fig. 7E, arrowheads). Finally as shown in Figure $7 F$, robust intensity-center shifts, representative of veritable $\mathrm{SCb}$ transport, were only possible when virtual interactions of cytosolic/SCb particles with vesicles were assumed (red data points), and a mere transit of vesicles through the axon (yellow data points) failed to generate sustained bulk movement of the virtual cytosolic particles. 
A PAGFP:synapsin/SyPhy:mRFP imaging (primary axon)

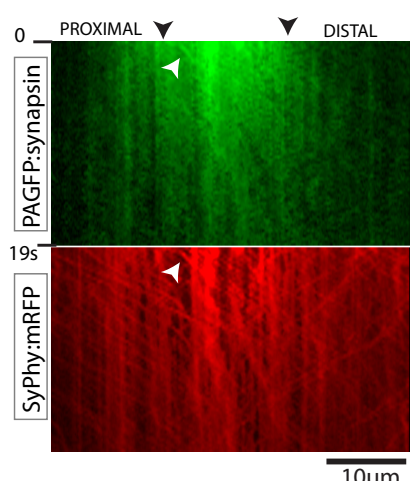

PROXIMAL

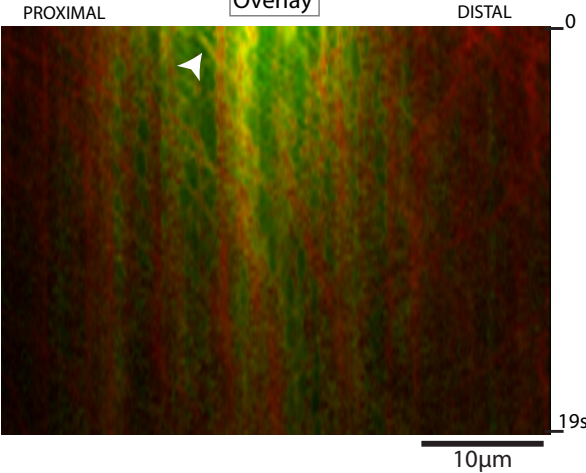

B

GFP:synapsin/SyPhy:mRFP imaging (distal axons)

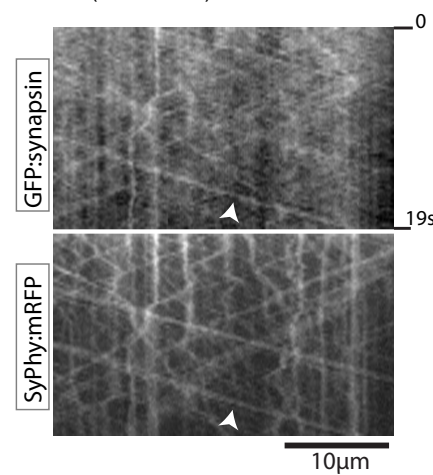

C GFP:synapsin/SyPhy:mRFP imaging (distal axons - contrast inverted)
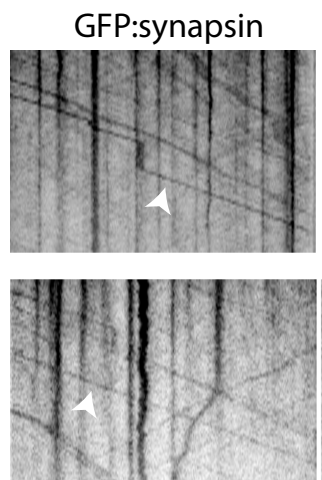

SyPhy:mRFP
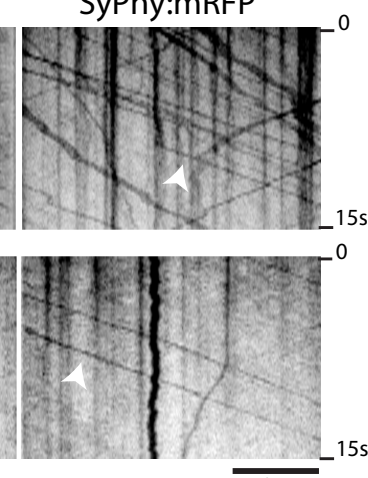

D Model of cytosolic SCb transport

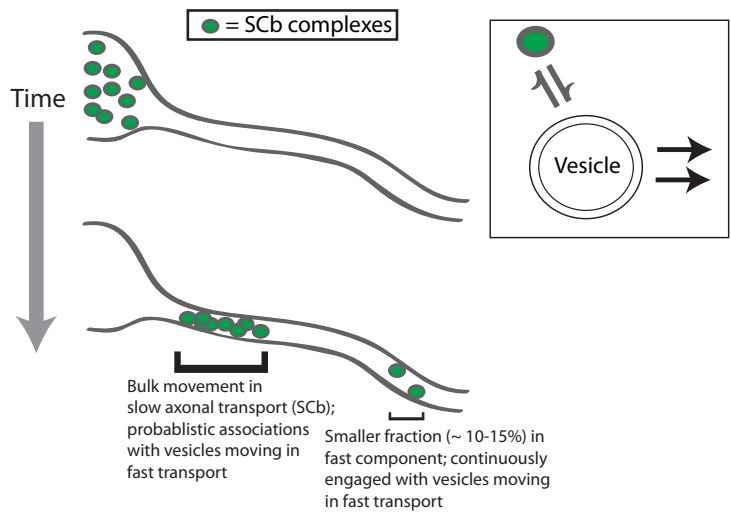

Figure 8. Cotransport of synapsin and synaptophysin in axons and a model. $\boldsymbol{A}$, Neurons were cotransfected with PAGFP:synapsin and synaptophysin:mRFP (SyPhy:mRFP); the PAGFP:synapsin was photoactivated in primary axons (black arrowheads indicate the photoactivated zone); and GFP/RFP fluorescence was simultaneously visualized by live imaging. There is colocalization of mobile synapsin particles with synaptophysin (white arrowhead indicates one such particle). B, Neurons were cotransfected with GFP:synapsin and SyPhy:mRFP, and GFP/RFP fluorescence was simultaneously visualized in thin, distal axons by live imaging (also see Results). Despite the diffuse fluorescence seen with GFP:synapsin, mobile synapsin particles are clearly seen over the background (top kymograph), and they colocalize with vesicles containing synaptophysin (bottom kymograph; white arrowheads indicate colocalized particle). C, Neurons were transfected and visualized as above. Selected kymographs show fortuitous instances in which axons were very thin and the diffuse glow was minimal, allowing clear visualization of synapsin/SyPhy particles (kymographs are contrast-inverted). Unequivocal instances of cotransport are seen (arrowheads). There is also colocalization in the majority of stationary particles (also see Discussion). D, A hypothetical model of $S C \mathrm{Cb}$ transport. Somatic cytosolic/soluble assemblies are transported into axons by probabilistic associations with vesicles moving in fast axonal transport. These associations are typically transient, and thus over time the bulk of cytosolic molecules is transported slowly in SCb. However, because of the stochastic nature of these associations, a small subset of cytosolic particles remain continuously associated with the vesicles and are thus seen in fast axonal transport.

These simulations predict that stochastic associations of cytosolic particles with persistently moving vesicles, sufficient to convey the bulk population with slow axonal transport rates, can also probabilistically generate individual particle kinetics that are much faster than that of the bulk population. Specifically, small subsets of cytosolic particles would be expected to move hundreds of microns ahead of the slowly moving bulk population. Thus, they favor a parsimonious model in which all observed SCb kinetics are related to differential associations of cytosolic cargoes with a common moving unit (vesicles), rather than one that requires distinct subpopulations of SCb cargoes (moving exclusively in fast or slow transport).

\section{Evidence for cotransport of synapsin particles with vesicles}

In previous experiments, we saw cotransport of synapsin particles with moving vesicles in thin distal axons with low background synapsin fluorescence (Roy et al., 2007, their Fig. 5; Scott et al., 2011, their Fig. 4), but without a conceptual framework, the interpretation was unclear. One possibility is that the rapidly mobile cytosolic particles seen in distal axons at steady state simply represent $\mathrm{SCb}$ cargoes that remain associated with vesicles for longer times, whereas visualization of SCb proteins using the photoactivation paradigm mainly highlights transient associations. Indeed, this seems to be the case. When we cotransfected neurons with PAGFP:synapsin and SyPhy:mRFP (to label moving vesicles) and simultaneously visualized the photoactivated synapsin and synaptophysin, we saw transient cotransport (Fig. $8 A$ ). However, when we transfected neurons with GFP:synapsin and synaptophysin:mRFP and visualized them simultaneously in thin distal axons (where such particles were discernible above the diffuse fluorescence), we saw unequivocal instances of cotransport (Fig. $8 B, C$ ). These direct observations lend further support to the hypothesis that associations with vesicles are critical for axonal transport of synapsin. Accordingly, we propose a model (Fig. $8 D$ ) in which, after synthesis, synapsin molecules assemble into multiprotein complexes that have affinity to membranes, and stochastically associate with mobile vesicles. Whereas a subset of cytosolic synapsin particles remain associated with vesicles for long periods and are conveyed in fast transport, the bulk population transiently interacts with moving vesicles and moves in slow axonal transport. 


\section{Discussion}

Previous pulse-chase radiolabeling studies have shown that soluble/cytosolic/SCb proteins synthesized in the soma are largely conveyed into axons by slow axonal transport. Closer examination of the individual profiles of radiolabeled "peaks" indicated that multiple SCb proteins appeared to move "coherently" (Garner and Lasek, 1982; for review, see Lasek et al., 1984). The cohesive transport of various soluble SCb proteins suggested that they were associating with a common structure/organelle (or "carrier") that was propelling them to their destinations (Lasek et al., 1984). However, until now, the identity of such a "carrier" has been a mystery. Our data here suggest that moving vesicles are such carriers for a classic SCb protein synapsin, and collectively support a model where slow transport of synapsin is mediated by intermittent and probabilistic associations of cytosolic assemblies with vesicles moving in fast axonal transport.

\section{Vesicles as "carriers" of soluble cargoes: links between fast and slow axonal transport}

In previous studies, we hypothesized the existence of "mobile units" that conveyed soluble protein assemblies via transient associations (Scott et al., 2011). Several lines of evidence now indicate that the former are "transport vesicles" moving in the fast component. First, conditions that attenuate vesicle transport also resulted in diminished synapsin transport (Fig. 4). Second, intrinsic fluctuations in fast vesicle transport had analogous effects on slow transport as well, implying that the two transport modalities are mechanistically coupled (Figs. 5 and $6 A-D$ ). Third, disrupting physical associations of synapsin with vesicles also inhibited its slow axonal transport (Fig. 6E), suggesting that these associations are mediating the transport. Finally, dynamic cotransport of synapsin particles with moving vesicles can be directly observed using both "pulse-chase" and steady-state imaging paradigms (Fig. 8; see the following discussion).

In hindsight, perhaps our proposed model is not entirely surprising. Radiolabeling studies have repeatedly revealed small pools of soluble/cytosolic proteins in fast components (Baitinger and Willard, 1987; Black et al., 1991; Petrucci et al., 1991; Mercken et al., 1995; Jensen et al., 1999; Susalka et al., 2000). However, in the absence of a logical framework for understanding slow transport, this small "fast-pool" has been largely ignored. Moreover, as mentioned above, we had also noticed cotransport of SCb particles with vesicles in live-cell assays when we imaged proteins at steady state (Roy et al., 2007; Scott et al., 2011). Cotransport of synapsin with vesicles in cultured neurons has also been reported (Ahmari et al., 2000). Interestingly, an earlier study using radiolabeled pulse-chase type paradigms in cultured sympathetic neurons grown within compartmentalized chambers reported a block in slow axonal transport of tubulin upon BFA incubation (Campenot et al., 2003). Although the authors focused on tubulin (moving in SCa), there were transport deficits of several other labeled proteins in their experiments, some of which are likely SCb proteins (based on the slow movement and molecular weights). Thus, reconciling all the available data and our simulations, a parsimonious view is that both SCb cargoes and membrane-anchoring proteins are conveyed via differential associations with vesicles.

It is intriguing that synapsin molecules seem to preferentially associate with anterogradely moving vesicles (Fig. 6). Such selective association of synapsin with anterogradely moving organelles is supported by previous studies using the "stop-flow" (or crush) approach to evaluate transport. In this paradigm, peripheral axons in vivo are crushed, and accumulation of endogenous proteins is evaluated in the proximal and distal segments by immunofluorescence. Interestingly, synapsin was found to selectively accumulate in the anterograde segments over time, whereas little, if any, accumulation was seen distally (for review, see Dahlström, 1992), and the authors speculated that perhaps synapsin was associated with anterogradely moving vesicles. Also, there are clear ultrastructural differences in the nature of anterogradely and retrogradely moving vesicles (Tsukita and Ishikawa, 1980). Supporting the general idea, radiolabeling studies also suggest that $\mathrm{SCb}$ proteins do not have significant retrograde components. However, the molecular basis of such selective association is unknown, and future studies may provide clarity.

\section{Axonal transport of soluble cargoes by "dynamic recruitment": a conceptual model}

Reconciling all the available experimental data and incorporating insights from the simulations, we posit the following scenario. After synthesis in free ribosomes, soluble/cytosolic proteins assemble into multiprotein complexes that can dynamically associate with vesicles. As vesicles move out of the soma, powered by motors, moving persistently, the soluble/cytosolic protein assemblies are also conveyed into the axon by virtue of their intermittent associations with the mobile vesicles. However, because such associations are dynamic and probabilistic, the overall flow of the cytosolic population is much slower than the overall movement of vesicles, giving rise to the two distinct populations (fast and slow) seen in radiolabeling experiments. Furthermore, because of the intermittent and probabilistic nature of such associations, a fraction of cytosolic molecules remains associated with mobile vesicles for long periods, resulting in persistent vectorial movements. This subset of persistent cytosolic particles likely represents the "fast-population" observed in previous radiolabeling studies of $\mathrm{SCb}$.

The above model helps explain a paradox that we have observed with synapsin and other SCb proteins. When we transfect neurons with SCb proteins tagged to PAGFP and visualize protein mobility in axons using our pulse-photoactivation paradigm, we see a slow anterogradely biased movement of the photoactivated SCb population (Scott et al., 2011; Tang et al., 2012a; current study). However, when we transfect neurons with $\mathrm{SCb}$ proteins tagged to conventional GFP and visualize thin (typically distal) axons, where the particles are resolvable over the diffuse GFP-glow invariably seen with soluble proteins, rapid vectorial particle-movement is seen (Fig. $8 B, C$ ) (Roy et al., 2007). Others have also reported similar results (Yang et al., 2010; Freundt et al., 2012). Despite this rapid vectorial movement of synapsin and other SCb proteins in cultured axons, overall, the $\mathrm{SCb}$ protein synapsin is clearly transported much more slowly, and with very distinct kinetics, than synaptophysin (Figs. 1 and 2 ), so there is little doubt that the overall transport of these proteins is distinct in cultured neurons, much like the in vivo situation. Indeed, our proposed model predicts a scenario where steady-state visualization of SCb (using conventional GFPtagged proteins) will bias one toward seeing the rapidly mobile particles, whereas the mobile soluble (slow) component would appear as a diffuse "haze" (see Fig. $8 B$ ).

An interesting aspect of SCb transport is the presence of stationary "hotspots" where the transported proteins cluster (e.g., Figs. $4 A$ and $8 A-C$, kymographs). These clusters colocalize with stationary vesicles as well (Fig. $8 C$ ). The mobile plume of synapsin also seems to concentrate at these clusters over time (Fig. $4 A$, left kymograph) (Scott et al., 2011; Tang et al., 2012b), suggesting that they may have a role in the transport process, perhaps by 
limiting the free diffusion of the protein (which would not be efficient enough to convey the molecules over long distances). The presence of vesicles in these clusters also provides the necessary means by which the soluble molecules could be transported, and further focused studies may resolve these issues.

Although free diffusion is a part of the overall behavior of soluble proteins, the microtubule/motor/ATP dependence of the motion seen in our studies clearly indicate an active process (Scott et al., 2011). Also, it is unlikely that the slow transport in our system is the result of axonal elongation resulting from advancing growth cones, as reported in some developing neurons (Suter and Miller, 2011). First, all experiments were performed on relatively older neurons with axons that were hundreds of microns long and had already formed en-passant boutons along distal axons (see Materials and Methods). As such, the ROIs imaged in the main axonal shafts were well away from putative growth cones. Besides, the collective evidence, including cotransport of cytosolic particles with vesicles, clearly implicates vesicle transport (and not axonal stretching/elongation) as the underlying mechanism.

\section{Experimental considerations}

The dynamics of synapsin and synaptophysin in our neurons are clearly very different ("biased flow" vs movement of individual vesicles), and the overall synapsin population moves at slow overall velocities resembling known $\mathrm{SCb}$ rates from radiolabeling studies (Fig. 1C) (Scott et al., 2011). Yet the ingress of synapsin into boutons is only approximately threefold slower than synaptophysin in our targeting experiments (Fig. $2 D, E$ ), whereas radiolabeling experiments predict a much larger difference. It is possible that the rapid transport rates of subpopulations of somatically derived synapsin (see above), the short distances we examined, and our method (quantifying total amounts reaching boutons) results in an overestimation of synapsin transport rates. Alternatively, our methods may also be underestimating synaptophysin transport rates, as this may be influenced by resident times and/or differential delivery of the protein into boutons. Nevertheless, the experiments do demonstrate that, despite having a common destination, the overall transport behavior of these two proteins is very different. Finally, previous studies have also shown that excessive amounts of synapsin can inhibit fast axonal transport in a phosphorylation-dependent manner (McGuinness et al., 1989). However, it is unlikely that this would be a factor in our studies, given the low levels of synapsin expression in our system, especially in experiments where the initial wave of newly synthesized synapsin is evaluated (Figs. 1 and 2). In any case, even if there was an effect of synapsin on vesicle transport in our system, it is difficult to imagine how that would affect the validity of our key results.

The extent to which our proposed mechanism applies to all $\mathrm{SCb}$ proteins remains unclear. Given the enormous diversity of $\mathrm{SCb}$, it is possible that many different transport mechanisms are at play. Indeed, a recent study proposed that mobility of the soluble protein of choline acetyltransferase in Drosophila neurons is mediated via a direct binding with kinesin-2 (Sadananda et al., 2012). Although further work is needed to address these issues, studies are now starting to unravel this enigmatic rate class that has been largely mysterious for decades.

\section{References}

Ahmari SE, Buchanan J, Smith SJ (2000) Assembly of presynaptic active zones from cytoplasmic transport packets. Nat Neurosci 3:445-451. CrossRef Medline
Baitinger C, Willard M (1987) Axonal transport of synapsin I-like proteins in rabbit retinal ganglion cells. J Neurosci 7:3723-3735. Medline

Black MM, Lasek RJ (1980) Slow components of axonal transport: two cytoskeletal networks. J Cell Biol 86:616-623. CrossRef Medline

Black MM, Chestnut MH, Pleasure IT, Keen JH (1991) Stable clathrin: uncoating protein (hsc70) complexes in intact neurons and their axonal transport. J Neurosci 11:1163-1172. Medline

Bourke GJ, El Alami W, Wilson SJ, Yuan A, Roobol A, Carden MJ (2002) Slow axonal transport of the cytosolic chaperonin CCT with Hsc73 and actin in motor neurons. J Neurosci Res 68:29-35. CrossRef Medline

Brady ST, Tytell M, Heriot K, Lasek RJ (1981) Axonal transport of calmodulin: a physiologic approach to identification of long-term associations between proteins. J Cell Biol 89:607-614. CrossRef Medline

Bray JJ, Fernyhough P, Bamburg JR, Bray D (1992) Actin depolymerizing factor is a component of slow axonal transport. J Neurochem 58:20812087. CrossRef Medline

Brown A (2003) Axonal transport of membranous and nonmembranous cargoes: a unified perspective. J Cell Biol 160:817-821. CrossRef Medline

Brown A, Wang L, Jung P (2005) Stochastic simulation of neurofilament transport in axons: the "stop-and-go" hypothesis. Mol Biol Cell 16:42434255. CrossRef Medline

Campenot RB, Soin J, Blacker M, Lund K, Eng H, MacInnis BL (2003) Block of slow axonal transport and axonal growth by brefeldin A in compartmented cultures of rat sympathetic neurons. Neuropharmacology 44: 1107-1117. CrossRef Medline

Cesca F, Baldelli P, Valtorta F, Benfenati F (2010) The synapsins: key actors of synapse function and plasticity. Prog Neurobiol 91:313-348. CrossRef Medline

Cheetham JJ, Hilfiker S, Benfenati F, Weber T, Greengard P, Czernik AJ (2001) Identification of synapsin I peptides that insert into lipid membranes. Biochem J 354:57-66. CrossRef Medline

Cheetham JJ, Murray J, Ruhkalova M, Cuccia L, McAloney R, Ingold KU, Johnston LJ (2003) Interaction of synapsin I with membranes. Biochem Biophys Res Commun 309:823-829. CrossRef Medline

Dahlström AB, Czernik AJ, Li JY (1992) Organelles in fast axonal transport: what molecules do they carry in anterograde vs retrograde directions, as observed in mammalian systems? Mol Neurobiol 6:157-177. CrossRef Medline

Dascher C, Balch WE (1994) Dominant inhibitory mutants of ARF1 block endoplasmic reticulum to Golgi transport and trigger disassembly of the Golgi apparatus. J Biol Chem 269:1437-1448. Medline

Denker A, Kröhnert K, Bückers J, Neher E, Rizzoli SO (2011) The reserve pool of synaptic vesicles acts as a buffer for proteins involved in synaptic vesicle recycling. Proc Natl Acad Sci U S A 108:17183-17188. CrossRef Medline

Dillman JF, III, Dabney LP, Pfister KK (1996) Cytoplasmic dynein is associated with slow axonal transport. Proc Natl Acad Sci U S A 93:141-144. CrossRef Medline

Dresbach T, Torres V, Wittenmayer N, Altrock WD, Zamorano P, Zuschratter W, Nawrotzki R, Ziv NE, Garner CC, Gundelfinger ED (2006) Assembly of active zone precursor vesicles: obligatory trafficking of presynaptic cytomatrix proteins Bassoon and Piccolo via a trans-Golgi compartment. J Biol Chem 281:6038-6047. CrossRef Medline

Elluru RG, Bloom GS, Brady ST (1995) Fast axonal transport of kinesin in the rat visual system: functionality of kinesin heavy chain isoforms. Mol Biol Cell 6:21-40. CrossRef Medline

Fletcher TL, Cameron P, De Camilli P, Banker G (1991) The distribution of synapsin I and synaptophysin in hippocampal neurons developing in culture. J Neurosci 11:1617-1626. Medline

Freundt EC, Maynard N, Clancy EK, Roy S, Bousset L, Sourigues Y, Covert M, Melki R, Kirkegaard K, Brahic M (2012) Neuron-to-neuron transmission of $\alpha$-synuclein fibrils through axonal transport. Ann Neurol 72:517524. CrossRef Medline

Garner JA, Lasek RJ (1982) Cohesive axonal transport of the slow component b complex of polypeptides. J Neurosci 2:1824-1835. Medline

Gitler D, Takagishi Y, Feng J, Ren Y, Rodriguiz RM, Wetsel WC, Greengard P, Augustine GJ (2004) Different presynaptic roles of synapsins at excitatory and inhibitory synapses. J Neurosci 24:11368-11380. CrossRef Medline

Jensen PH, Li JY, Dahlström A, Dotti CG (1999) Axonal transport of synucleins is mediated by all rate components. Eur J Neurosci 11:33693376. CrossRef Medline 
Lasek RJ, Garner JA, Brady ST (1984) Axonal transport of the cytoplasmic matrix. J Cell Biol 99:212s-221s. CrossRef Medline

Lorenz T, Willard M (1978) Subcellular fractionation of intra-axonally transport polypeptides in the rabbit visual system. Proc Natl Acad Sci U S A 75:505-509. CrossRef Medline

Lund LM, McQuarrie IG (2001) Calcium/calmodulin-dependent protein kinase IIalpha in optic axons moves with slow axonal transport and undergoes posttranslational modification. Biochem Biophys Res Commun 289:1157-1161. CrossRef Medline

McGuinness TL, Brady ST, Gruner JA, Sugimori M, Llinas R, Greengard P (1989) Phosphorylation-dependent inhibition by synapsin I of organelle movement in squid axoplasm. J Neurosci 9:4138-4149. Medline

Menegon A, Bonanomi D, Albertinazzi C, Lotti F, Ferrari G, Kao HT, Benfenati, Baldelli P, Valtorta F (2006) Protein kinase A-mediated synapsin I phosphorylation is a central modulator of $\mathrm{Ca} 2+$-dependent synaptic activity. J Neurosci 26, 11670-11681. CrossRef Medline

Mercken M, Fischer I, Kosik KS, Nixon RA (1995) Three distinct axonal transport rates for tau, tubulin, and other microtubule-associated proteins: evidence for dynamic interactions of tau with microtubules in vivo. J Neurosci 15:8259-8267. Medline

Paggi P, Petrucci TC (1992) Neuronal compartments and axonal transport of synapsin I. Mol Neurobiol 6:239-251. CrossRef Medline

Petrucci TC, Macioce P, Paggi P (1991) Axonal transport kinetics and posttranslational modification of synapsin I in mouse retinal ganglion cells. J Neurosci 11:2938-2946. Medline

Roy S, Coffee P, Smith G, Liem RK, Brady ST, Black MM (2000) Neurofilaments are transported rapidly but intermittently in axons: implications for slow axonal transport. J Neurosci 20:6849-6861. Medline

Roy S, Winton MJ, Black MM, Trojanowski JQ, Lee VM (2007) Rapid and intermittent cotransport of slow component-b proteins. J Neurosci 27: 3131-3138. CrossRef Medline

Roy S, Yang G, Tang Y, Scott DA (2011) A simple photoactivation and image analysis module for visualizing and analyzing axonal transport with high temporal resolution. Nat Protoc 7:62-68. CrossRef Medline

Sadananda A, Hamid R, Doodhi H, Ghosal D, Girotra M, Jana SC, Ray K (2012) Interaction with a kinesin-2 tail propels choline acetyltransferase flow towards synapse. Traffic 13:979-991. CrossRef Medline

Scott DA, Das U, Tang Y, Roy S (2011) Mechanistic logic underlying the axonal transport of cytosolic proteins. Neuron 70:441-454. CrossRef Medline

Susalka SJ, Hancock WO, Pfister KK (2000) Distinct cytoplasmic dynein complexes are transported by different mechanisms in axons. Biochim Biophys Acta 1496:76-88. CrossRef Medline

Suter DM, Miller KE (2011) The emerging role of forces in axonal elongation. Prog Neurobiol 94:91-101. CrossRef Medline

Tang Y, Das U, Scott DA, Roy S (2012a) The slow axonal transport of alphasynuclein-mechanistic commonalities amongst diverse cytosolic cargoes. Cytoskeleton (Hoboken) 69:506-513. CrossRef Medline

Tang Y, Scott DA, Das U, Edland SD, Radomski K, Koo EH, Roy S (2012b) Early and selective impairments in axonal transport kinetics of synaptic cargoes induced by soluble amyloid $\beta$-protein oligomers. Traffic 13:681693. CrossRef Medline

Tsukita S, Ishikawa H (1980) The movement of membranous organelles in axons: electron microscopic identification of anterogradely and retrogradely transported organelles. J Cell Biol 84:513-530. CrossRef Medline

Tsuriel S, Geva R, Zamorano P, Dresbach T, Boeckers T, Gundelfinger ED, Garner CC, Ziv NE (2006) Local sharing as a predominant determinant of synaptic matrix molecular dynamics. PLoS Biol 4:e271. CrossRef Medline

Vale RD (2003) The molecular motor toolbox for intracellular transport. Cell 112:467-480. CrossRef Medline

Verhey KJ, Hammond JW (2009) Traffic control: regulation of kinesin motors. Nat Rev Mol Cell Biol 10:765-777. CrossRef Medline

Wang L, Ho CL, Sun D, Liem RK, Brown A (2000) Rapid movement of axonal neurofilaments interrupted by prolonged pauses. Nat Cell Biol 2:137-141. CrossRef Medline

Yang ML, Hasadsri L, Woods WS, George JM (2010) Dynamic transport and localization of alpha-synuclein in primary hippocampal neurons. Mol Neurodegener 5:9. CrossRef Medline

Yuan A, Mills RG, Bamburg JR, Bray JJ (1999) Cotransport of glyceraldehyde-3-phosphate dehydrogenase and actin in axons of chicken motoneurons. Cell Mol Neurobiol 19:733-744. CrossRef Medline

Zahn TR, Angleson JK, MacMorris MA, Domke E, Hutton JF, Schwartz C, Hutton JC (2004) Dense core vesicle dynamics in Caenorhabditis elegans neurons and the role of kinesin UNC-104. Traffic 5:544-559. CrossRef Medline 